\title{
Municipal Waste Plastic Conversion into Different Category of Liquid Hydrocarbon Fuel
}

\author{
Moinuddin Sarker \\ Natural States Research, Inc. \\ USA
}

\section{Introduction}

Plastics were first invented in 1860, but have only been widely used in the last 30 years. Plastics are light, durable, modifiable and hygienic. Plastics are made up of long chain of molecules called polymers. Polymers are made when naturally occurring substances such as crude oil or petroleum are transformed into other substances with completely different properties. These polymers can then be made into granules, powders and liquids, becoming raw materials for plastic products. Worldwide plastics production increases 80 million tons every year. Global production and consumption of plastics have increased, from less than 5 million tons in the year 1950 to 260 million tons in the year 2007. Of those over one third is being used for packaging, while rest is used for other sectors. Plastic production has increased by more than $500 \%$ over the past 30 years. Per capita consumption of plastics will increase by more than $50 \%$ during the next decades. In the Western Europe total annual household waste generation is approximately $500 \mathrm{~kg}$ per capita and $750 \mathrm{~kg}$ per capita in the United States; $12 \%$ of this total waste is plastics. The global total waste plastic generation is estimated to be over 210 million tons per year. US alone generate 48 million tons per year (Stat data from EPA). The growth in plastics use is due to their beneficial characteristics; $21^{\text {st }}$ century Economic growth making them even more suitable for a wide variety of applications, such as: food and product packaging, car manufacturing, agricultural use, housing products and etc. Because of good safety and hygiene properties for food packaging, excellent thermal and electrical insulation properties, plastics are more desirable among consumers. Low production cost, lower energy consumption and $\mathrm{CO}_{2}$ emissions during production of plastics are relatively lower than making alternative materials, such as glass, metals and etc. Yet for all their advantages, plastics have a considerable downside in terms of their environmental impact. Plastic production requires large amounts of resources, primarily fossil fuels and $8 \%$ of the world's annual oil production is used in the production of plastics. Potentially harmful chemicals are added as stabilizers or colorants. Many of these have not undergone environmental risk assessment and their impact on human health and environment is currently uncertain. Worldwide municipal sites like shops or malls had the largest proportion of plastic rubbish items. Ocean soup swirling the debris of plastics trash in the Pacific Ocean has now grown to a size that is twice as large as the continental US. In 2006, 11.5 million of tons of plastics were wasted in the landfill. These types of disposal of the waste plastics release toxic gas; which has negative impact on environment. 
Most plastics are non-biodegradable and they take long time to break down in landfill, estimated to be more than a century. Plastic waste also has a detrimental impact on wild life; plastic waste in the oceans is estimated to cause the death of more than a million seabirds and more than 100,000 marine mammals every year (UN Environmental Program Estimate). Along with this hundreds of thousands of sea turtles, whales and other marine mammals die every year eating discarded waste plastic bags mistaken for food. Setting up intermediate treatment plants for waste plastic, such as: plastic incineration, recycle, or obtaining the landfill for reclamation is difficult. The types of the waste plastics are LDPE, HDPE, PP, PS, PVC, PETE, PLA and etc. The problems of waste plastics can't be solved by landfilling or incineration, because the safety deposits are expensive and incineration stimulates the growing emission of harmful green house gases, e.g COx, NOx, SOx and etc. By using NSR's new technology we can convert all types of waste plastics into liquid hydrocarbon fuel by setting temperature profile 370 degree $C$ to 420 degree $C$, we can resolve all waste plastic problems including land, ocean, river and green house effects. Many of researcher and experts have done a lot of research and work on waste plastics; some of the thesis's are on thermal degradation process [1-10], pyrolysis process [11-20] and catalytic conversion process [21-30]. Producing fuels can be alternative of heating oil, gasoline, naphtha, aviation, diesel and fuel oil. We also produce light gaseous (natural gas) hydrocarbon compound $\left(\mathrm{C}_{1}-\mathrm{C}_{4}\right)$, such as: methane, ethane, propane and butane. This process is profitable because it requires less production cost per gallon. We can produce individual plastic to fuel, mixed waste plastic to fuel and that produced fuel can make different category fuels by using further fractional distillation process. This NSR technology will not only reduce the production cost of fuel, but it will also reduce $9 \%$ of foreign oil dependency, create more electricity and new jobs all over the world. To mitigate the present world market demand, we can substitute this method as a potential source of new renewable energy.

\section{Experimental section}

\subsection{Waste plastics properties}

A plastic has physical and chemical properties. Different types of plastics displayed distinguishable characteristics and properties. Many kinds of plastics are appeared like LDPE, HDPE, PP, PS, PVC \&PETE etc. Several individual plastics properties are elaborated in shortly, that's given below in Table-1, Table-2, Table-3 and Table-4.

\begin{tabular}{ccc}
\hline Quantity & Value & Units \\
\hline Thermal expansion & $110-130$ & $\mathrm{e}-6 / \mathrm{K}$ \\
Thermal conductivity & $0.46-0.52$ & $\mathrm{~W} / \mathrm{m} . \mathrm{K}$ \\
Specific heat & $1800-2700$ & $\mathrm{~J} / \mathrm{kg} . \mathrm{K}$ \\
Melting temperature & $108-134$ & ${ }^{\circ} \mathrm{C}$ \\
Glass temperature & $-110--110$ & ${ }^{\circ} \mathrm{C}$ \\
Service temperature & $-30-85$ & ${ }^{\circ} \mathrm{C}$ \\
Density & $940-965$ & $\mathrm{~kg} / \mathrm{m}^{3}$ \\
Resistivity & $5 \mathrm{e}+17-1 \mathrm{e}+21$ & $\mathrm{Ohm} . \mathrm{mm}^{2} / \mathrm{m}$ \\
Shrinkage & $2-4$ & $\%$ \\
Water absorption & $0.01-0.01$ & $\%$ \\
\hline
\end{tabular}

Table 1. HDPE-2 Plastic Properties 


\begin{tabular}{ccc}
\hline Quantity & Value & Units \\
\hline Thermal expansion & $150-200$ & $\mathrm{e}-6 / \mathrm{K}$ \\
Thermal conductivity & $0.3-0.335$ & $\mathrm{~W} / \mathrm{m} . \mathrm{K}$ \\
Specific heat & $1800-3400$ & $\mathrm{~J} / \mathrm{kg} . \mathrm{K}$ \\
Melting temperature & $125-136$ & ${ }^{\circ} \mathrm{C}$ \\
Glass temperature & $-110--110$ & ${ }^{\circ} \mathrm{C}$ \\
Service temperature & $-30-70$ & ${ }^{\circ} \mathrm{C}$ \\
Density & $910-928$ & $\mathrm{~kg} / \mathrm{m}^{3}$ \\
Resistivity & $5 \mathrm{e}+17-1 \mathrm{e}+21$ & $\mathrm{Ohm} . \mathrm{mm}^{2} / \mathrm{m}$ \\
Breakdown potential & $17.7-39.4$ & $\mathrm{kV} / \mathrm{mm}^{\%}$ \\
Shrinkage & $1.5-3$ & $\%$ \\
Water absorption & $0.005-0.015$ &
\end{tabular}

Table 2. LDPE-4 Plastic Properties

\begin{tabular}{ccc}
\hline Quantity & Value & Units \\
\hline Thermal expansion & $180-180$ & $\mathrm{e}-6 / \mathrm{K}$ \\
Thermal conductivity & $0.22-0.22$ & $\mathrm{~W} / \mathrm{m} \cdot \mathrm{K}$ \\
Melting temperature & $160-165$ & ${ }^{\circ} \mathrm{C}$ \\
Glass temperature & $-10--10$ & ${ }^{\circ} \mathrm{C}$ \\
Service temperature & $-10-110$ & ${ }^{\circ} \mathrm{C}$ \\
Density & $902-907$ & $\mathrm{~kg} / \mathrm{m}^{3}$ \\
Resistivity & $5 \mathrm{e}+21-1 \mathrm{e}+22$ & $\mathrm{Ohm} \cdot \mathrm{mm}^{2} / \mathrm{m}$ \\
Breakdown potential & $55-90$ & $\mathrm{kV} / \mathrm{mm}$ \\
Shrinkage & $0.8-2$ & $\%$ \\
\hline
\end{tabular}

Table 3. PP-5 Plastic Properties

\begin{tabular}{ccc}
\hline Quantity & Value & Units \\
\hline Thermal expansion & $60-80$ & $\mathrm{e}-6 / \mathrm{K}$ \\
Thermal conductivity & $0.14-0.16$ & $\mathrm{~W} / \mathrm{m} . \mathrm{K}$ \\
Specific heat & $1300-1300$ & $\mathrm{~J} / \mathrm{kg} \cdot \mathrm{K}$ \\
Glass temperature & $80-98$ & ${ }^{\circ} \mathrm{C}$ \\
Service temperature & $-10-90$ & ${ }^{\circ} \mathrm{C}$ \\
Density & $1040-1050$ & $\mathrm{~kg} / \mathrm{m}^{3}$ \\
Resistivity & $1 \mathrm{e}+22-1 \mathrm{e}+22$ & $\mathrm{Ohm} \cdot \mathrm{mm}^{2} / \mathrm{m}$ \\
Breakdown potential & $100-160$ & $\mathrm{kV} / \mathrm{mm}^{\circ}$ \\
Shrinkage & $0.3-0.7$ & $\%$ \\
\hline
\end{tabular}

Table 4. PS-6 Plastic Properties

\subsection{Pre analysis of Gas Chromatography \& Mass Spectrometer (GC/MS) analysis}

Before starting the fuel production experiment, we have analyzed each of the individual raw waste plastics. Types of analyzed raw waste plastics are following, HDPE-2 (High Density Polyethylene), LDPE-4 (Low Density Polyethylene), PP-5 (Polypropylene) and PS-6 (Polystyrene). 


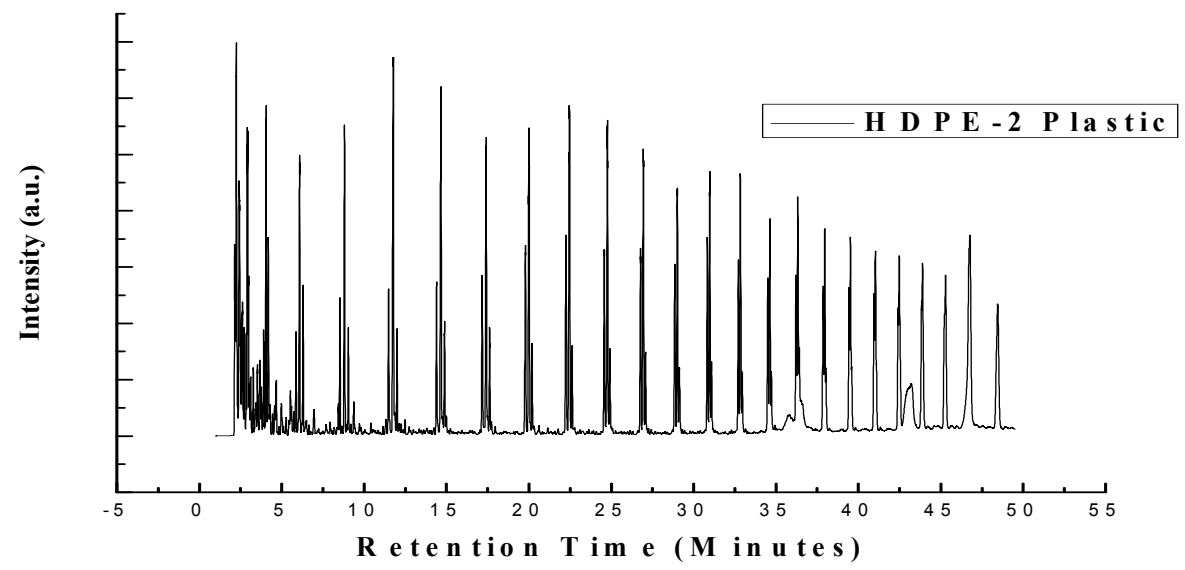

Fig. 1. GC/MS Chromatogram of HDPE-2 Raw Waste Plastic

\begin{tabular}{|c|c|c|c|c|c|}
\hline $\begin{array}{c}\text { Retention } \\
\text { Time } \\
\end{array}$ & $\begin{array}{c}\text { Compound } \\
\text { Name }\end{array}$ & Formula & $\begin{array}{c}\text { Retention } \\
\text { Time }\end{array}$ & $\begin{array}{c}\text { Compound } \\
\text { Name }\end{array}$ & Formula \\
\hline 2.14 & Propane & $\mathrm{C}_{3} \mathrm{H}_{8}$ & 22.62 & Tetradecane & $\mathrm{C}_{14} \mathrm{H}_{30}$ \\
\hline 2.23 & 3-Butyn-1-ol & $\mathrm{C}_{4} \mathrm{H}_{6} \mathrm{O}$ & 24.57 & $\begin{array}{c}1,13- \\
\text { Tetradecadiene }\end{array}$ & $\mathrm{C}_{14} \mathrm{H}_{26}$ \\
\hline 17.61 & Dodecane & $\mathrm{C}_{12} \mathrm{H}_{26}$ & 40.94 & 1,19-Eicosadiene & $\mathrm{C}_{20} \mathrm{H}_{38}$ \\
\hline 19.78 & $\begin{array}{c}\text { 1,13- } \\
\text { Tetradecadiene }\end{array}$ & $\mathrm{C}_{14} \mathrm{H}_{26}$ & 41.02 & 1-Docosene & $\mathrm{C}_{22} \mathrm{H}_{44}$ \\
\hline 20.00 & 1-Tridecene & $\mathrm{C}_{13} \mathrm{H}_{26}$ & 42.48 & 1-Docosene & $\mathrm{C}_{22} \mathrm{H}_{44}$ \\
\hline 20.19 & Tridecane & $\mathrm{C}_{13} \mathrm{H}_{28}$ & 43.89 & 1-Tetracosanol & $\mathrm{C}_{24} \mathrm{H}_{50} \mathrm{O}$ \\
\hline 22.24 & $\begin{array}{c}\text { 1,13- } \\
\text { Tetradecadiene }\end{array}$ & $\mathrm{C}_{14} \mathrm{H}_{26}$ & 45.28 & 9-Tricosene, (Z)- & $\mathrm{C}_{23} \mathrm{H}_{46}$ \\
\hline 22.45 & Cyclotetradecane & $\mathrm{C}_{14} \mathrm{H}_{28}$ & 46.76 & $\begin{array}{c}17- \\
\text { Pentatriacontene }\end{array}$ & $\mathrm{C}_{35} \mathrm{H}_{70}$ \\
\hline
\end{tabular}

Table 4. GC/MS Compound List of HDPE-2 Waste Plastic 


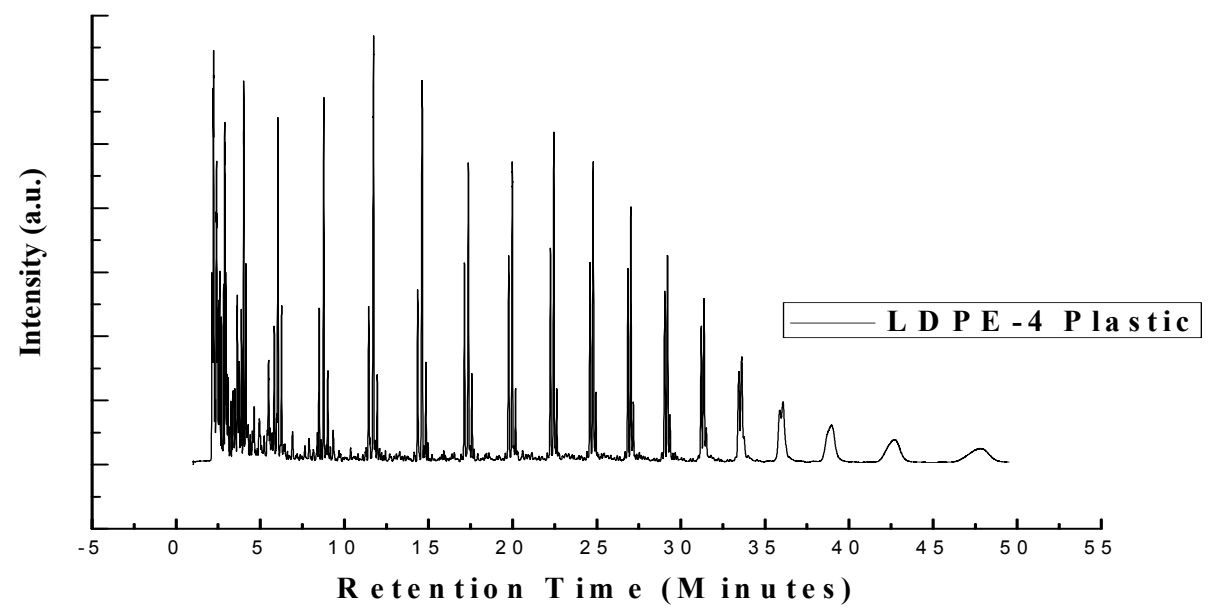

Fig. 2. GC/MS Chromatogram of LDPE-4 Raw Waste Plastic

\begin{tabular}{|c|c|c|c|c|c|}
\hline $\begin{array}{l}\text { Retention } \\
\text { Time } \\
\text { (Minutes) }\end{array}$ & $\begin{array}{l}\text { Compound } \\
\text { Name }\end{array}$ & Formula & $\begin{array}{l}\text { Retention } \\
\text { Time } \\
\text { (Minutes) }\end{array}$ & $\begin{array}{c}\text { Compound } \\
\text { Name }\end{array}$ & Formula \\
\hline 2.11 & Propane & $\mathrm{C}_{3} \mathrm{H}_{8}$ & 17.13 & $\begin{array}{c}1,11- \\
\text { Dodecadiene }\end{array}$ & $\mathrm{C}_{12} \mathrm{H}_{22}$ \\
\hline 2.19 & $\begin{array}{l}\text { Cyclopropyl } \\
\text { carbinol }\end{array}$ & $\mathrm{C}_{4} \mathrm{H}_{8} \mathrm{O}$ & 17.37 & Cyclododecane & $\mathrm{C}_{12} \mathrm{H}_{24}$ \\
\hline 11.44 & 1,9-Decadiene & $\mathrm{C}_{10} \mathrm{H}_{18}$ & 33.62 & 1-Nonadecene & $\mathrm{C}_{19} \mathrm{H}_{38}$ \\
\hline 11.73 & Cyclodecane & $\mathrm{C}_{10} \mathrm{H}_{20}$ & & & \\
\hline 11.95 & Decane & $\mathrm{C}_{10} \mathrm{H}_{22}$ & 35.87 & $\begin{array}{c}1,19- \\
\text { Eicosadiene }\end{array}$ & $\mathrm{C}_{20} \mathrm{H}_{38}$ \\
\hline 14.35 & $\begin{array}{c}1,10- \\
\text { Undecadiene }\end{array}$ & $\mathrm{C}_{11} \mathrm{H}_{20}$ & 36.08 & $\begin{array}{l}\text { 1-Heneicosyl } \\
\text { formate }\end{array}$ & $\mathrm{C}_{22} \mathrm{H}_{44} \mathrm{O}_{2}$ \\
\hline 14.61 & 1-Undecene & $\mathrm{C}_{11} \mathrm{H}_{22}$ & 42.76 & 1-Docosanol & $\mathrm{C}_{22} \mathrm{H}_{46} \mathrm{O}$ \\
\hline 14.84 & Undecane & $\mathrm{C}_{11} \mathrm{H}_{24}$ & 47.91 & $\begin{array}{l}\text { 9-Tricosene, } \\
\text { (Z)- }\end{array}$ & $\mathrm{C}_{23} \mathrm{H}_{46}$ \\
\hline
\end{tabular}

Table 5. GC/MS Chromatogram Compound list of LDPE-4 Raw Waste Plastic 


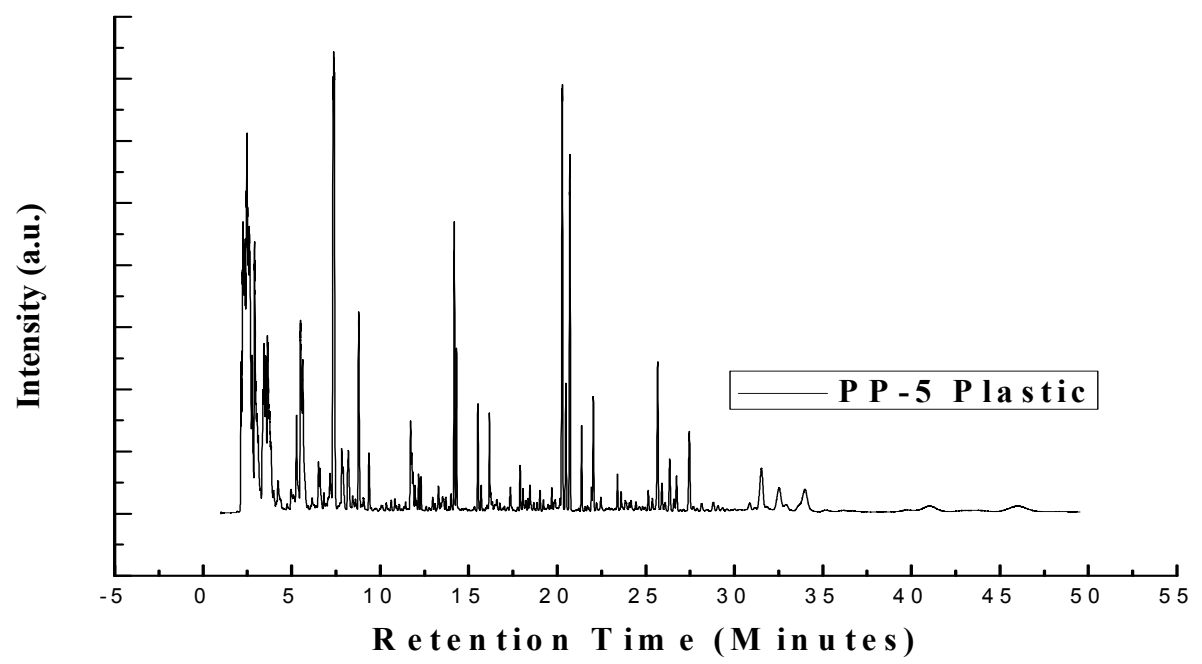

Fig. 3. GC/MS Chromatogram of PP-5 Raw Waste Plastic

\begin{tabular}{|c|c|c|c|c|c|}
\hline $\begin{array}{c}\text { Retention } \\
\text { Time } \\
\text { (Minutes) }\end{array}$ & Compound Name & Formula & $\begin{array}{c}\text { Retention } \\
\text { Time } \\
\text { (Minutes) }\end{array}$ & $\begin{array}{l}\text { Compound } \\
\text { Name }\end{array}$ & Formula \\
\hline 2.13 & Cyclopropane & $\mathrm{C}_{3} \mathrm{H}_{6}$ & 12.29 & $\begin{array}{l}\text { Decane, 4- } \\
\text { methyl- }\end{array}$ & $\mathrm{C}_{11} \mathrm{H}_{24}$ \\
\hline 2.26 & 1-Butyne & $\mathrm{C}_{4} \mathrm{H}_{6}$ & 14.18 & $\begin{array}{l}\text { 2-Dodecene, } \\
\text { (E)- }\end{array}$ & $\mathrm{C}_{12} \mathrm{H}_{24}$ \\
\hline 9.36 & $\begin{array}{l}\text { 1,6-Octadiene, 2,5- } \\
\text { dimethyl-, (E)- }\end{array}$ & $\mathrm{C}_{10} \mathrm{H}_{18}$ & 26.35 & $\begin{array}{c}\text { 1-Hexadecanol, } \\
3,7,11,15- \\
\text { tetramethyl- }\end{array}$ & $\mathrm{C}_{20} \mathrm{H}_{42} \mathrm{O}$ \\
\hline 11.71 & $\begin{array}{c}\text { Nonane, 2-methyl-3- } \\
\text { methylene- }\end{array}$ & $\mathrm{C}_{11} \mathrm{H}_{22}$ & 31.52 & $\begin{array}{l}\text { 1-Heneicosyl } \\
\text { formate }\end{array}$ & $\mathrm{C}_{22} \mathrm{H}_{44} \mathrm{O}_{2}$ \\
\hline 11.78 & $\begin{array}{l}\text { 1-Ethyl-2,2,6- } \\
\text { trimethylcyclohexane }\end{array}$ & $\mathrm{C}_{11} \mathrm{H}_{22}$ & 32.51 & 1-Nonadecanol & $\mathrm{C}_{19} \mathrm{H}_{40} \mathrm{O}$ \\
\hline 12.17 & Nonane, 2,6-dimethyl- & $\mathrm{C}_{11} \mathrm{H}_{24}$ & 33.98 & $\begin{array}{c}1,22- \\
\text { Docosanediol }\end{array}$ & $\mathrm{C}_{22} \mathrm{H}_{46} \mathrm{O}_{2}$ \\
\hline
\end{tabular}

Table 6. GC/MS Chromatogram Compound List of PP-5 Raw Waste Plastic 


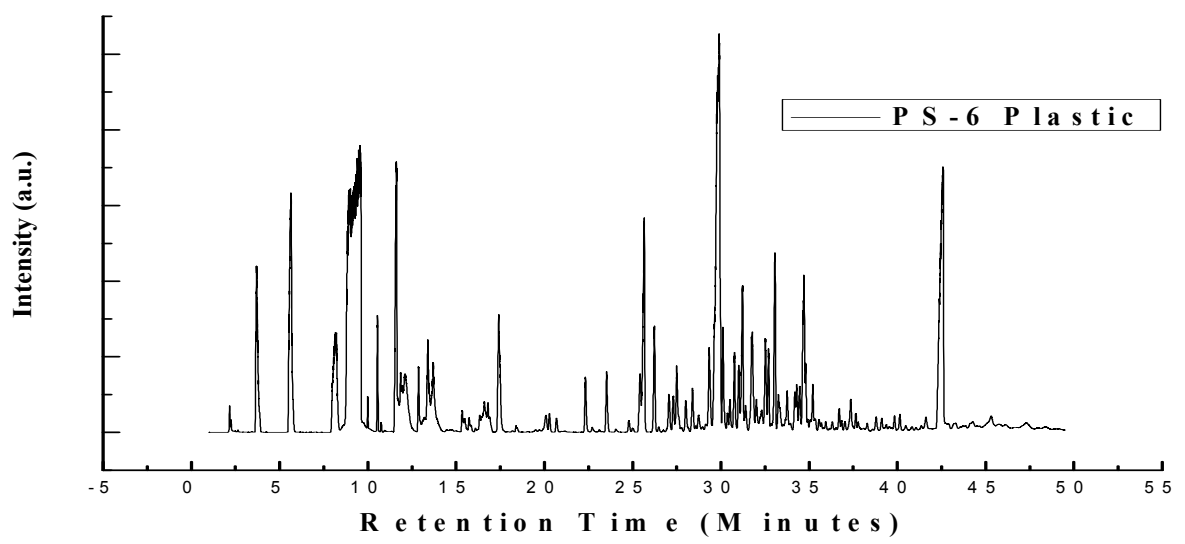

Fig. 4. GC/MS Chromatogram of PS-6 Raw Waste Plastic

\begin{tabular}{|c|c|c|c|c|c|}
\hline $\begin{array}{l}\text { Retention } \\
\text { Time } \\
\text { (Minutes) }\end{array}$ & Compound Name & Formula & $\begin{array}{l}\text { Retention } \\
\text { Time } \\
\text { (Minutes) }\end{array}$ & Compound Name & Formula \\
\hline 2.17 & Cyclopropane & $\mathrm{C}_{3} \mathrm{H}_{6}$ & 24.78 & 1,1'-Biphenyl, 3-methyl- & $\mathrm{C}_{13} \mathrm{H}_{12}$ \\
\hline 2.24 & $\begin{array}{l}\text { Methylenecyclopro- } \\
\text { pane }\end{array}$ & $\mathrm{C}_{4} \mathrm{H}_{6}$ & 25.64 & 1,2-Diphenylethylene & $\mathrm{C}_{14} \mathrm{H}_{12}$ \\
\hline 5.52 & $\begin{array}{l}\text { Toluene } \\
\text { 1,4- }\end{array}$ & $\mathrm{C}_{7} \mathrm{H}_{8}$ & 27.30 & 1,2-Diphenylcyclopropane & $\mathrm{C}_{15} \mathrm{H}_{14}$ \\
\hline 20.09 & $\begin{array}{c}\text { Methanonaphthalene, } \\
\text { 1,4-dihydro- }\end{array}$ & $\mathrm{C}_{11} \mathrm{H}_{10}$ & 37.35 & $\begin{array}{l}\text { Naphthalene, 1- } \\
\text { (phenylmethyl)- }\end{array}$ & $\mathrm{C}_{17} \mathrm{H}_{14}$ \\
\hline 20.28 & $\begin{array}{l}\text { Benzocyclohepta- } \\
\text { triene }\end{array}$ & $\mathrm{C}_{11} \mathrm{H}_{10}$ & 37.63 & p-Terphenyl & $\mathrm{C}_{18} \mathrm{H}_{14}$ \\
\hline 20.67 & $\begin{array}{l}\text { Naphthalene, 1- } \\
\text { methyl- }\end{array}$ & $\mathrm{C}_{11} \mathrm{H}_{10}$ & 38.79 & Fluoranthene, 2-methyl- & $\mathrm{C}_{17} \mathrm{H}_{12}$ \\
\hline 22.32 & Biphenyl & $\mathrm{C}_{12} \mathrm{H}_{10}$ & 39.83 & $\begin{array}{c}\text { Benzene, 1,1'-[1- } \\
\text { (ethylthio)propylidene]bis- }\end{array}$ & $\mathrm{C}_{17} \mathrm{H}_{20} \mathrm{~S}$ \\
\hline 23.52 & Diphenylmethane & $\mathrm{C}_{13} \mathrm{H}_{12}$ & 40.13 & $\begin{array}{c}\text { Benzene, 1,1',1",1"'-(1,2,3,4- } \\
\text { butanetetrayl)tetrakis- }\end{array}$ & $\mathrm{C}_{28} \mathrm{H}_{26}$ \\
\hline
\end{tabular}

Table 7. GC/MS Chromatogram of PS-6 Raw Waste Plastic Compound List 
Individual raw waste plastics of GCMS pre-analysis in accordance with their numerous retention times many compound are found, some of them are mentioned shortly. In HDPE-2 raw waste plastics on retention time 2.14, compound is Propane $\left(\mathrm{C}_{3} \mathrm{H}_{8}\right)$, on retention time 22.45, compound is Cyclotetradecane and finally on retention time 46.76 obtained compound is Pentatriacotene $\left(\mathrm{C}_{35} \mathrm{H}_{70}\right)$ [Shown above Fig.1 and Table-4]. In LDPE-4 raw waste plastics on retention time 2.11, compound is Propane $\left(\mathrm{C}_{3} \mathrm{H}_{8}\right)$, on retention time 14.84, compound is Undecane $\left(\mathrm{C}_{11} \mathrm{H}_{24}\right)$ and finally on retention time 47.91 obtained compound is 9Tricosene $(\mathrm{Z})-\left(\mathrm{C}_{23} \mathrm{H}_{46}\right)$ [Shown above Fig.2 and Table-5]. In PP-5 initially on retention time 2.13 compound is Cyclopropane $\left(\mathrm{C}_{3} \mathrm{H}_{6}\right)$ and finally on retention time 33.98 obtained compound is 1, 22-Docosanediol $\left(\mathrm{C}_{22} \mathrm{H}_{46} \mathrm{O}_{2}\right)$ [Shown above Fig.3 and Table-6]. Accordingly in PS-6 on retention time 2.17 found compound is Cyclopropane and eventually on retention time 40.13 obtained compound is Benzene, 1,1',1",1"'-(1,2,3,4-butanetetryl)tetrakis[Shown above Fig.4 and Table-7].

\subsection{Sample preparation}

We take municipal mixed waste plastics or any other source of mixed waste plastics; we initially sort out the foreign particles, clean the waste plastics and clean wash them with detergent. After clean up all waste plastics spread in the open air for air dry. When dried out we shred them by scissors, now shredded plastics are grinded by grinding machine. Grinded samples structure are granular form small particles and that easy to put into the reactor. In our laboratory facility we can utilize $400 \mathrm{~g}$ to $3 \mathrm{~kg}$ of grinding sample for any experimental purposes.

\section{Process description}

\subsection{Individual plastic to fuel production process}

The process has been conducted in small scales with individual plastics in laboratory, on various waste plastics types; High-density polyethylene (HDPE, code 2), low-density polyethylene (LDPE, code 4), polypropylene (PP, code 5) and polystyrene (PS, code 6). These plastic types were investigated singly. For small-scale laboratory process the weight of input waste plastics ranges from 400 grams to $3 \mathrm{~kg}$. These waste plastics are collected, optionally sorted, cleaned of contaminants, and shredded into small pieces prior to the thermal liquefaction process. The process of converting the waste plastic to alternative energy begins with heating the solid plastic with or without the presence of cracking catalyst to form liquid slurry (thermal liquefaction in the range of $370-420^{\circ} \mathrm{C}$ ), condensing the vapor with standard condensing column to form liquid hydrocarbon fuel termed "NSR fuel". Preliminary tests on the produced NSR fuel have shown that it is a mixture of various hydrocarbons range. The produced fuel density varies based on individual plastic types. In equivalent to obtaining the liquid hydrocarbon fuel we also receive light gaseous hydrocarbon compounds $\left(C_{1}-C_{4}\right)$ which resembles natural gas. Further fractional distillation based on different temperature is producing different category fuels; such as heating oil, gasoline, Naphtha (chemical), Aviation, Diesel and Fuel Oil. Experiment diagram given below in Fig.5.

\subsection{Mixed waste plastic to fuel production process}

Mixed waste plastics to fuel production process performed in the laboratory on various waste plastics types; High-density polyethylene (HDPE, code 2), low-density polyethylene 


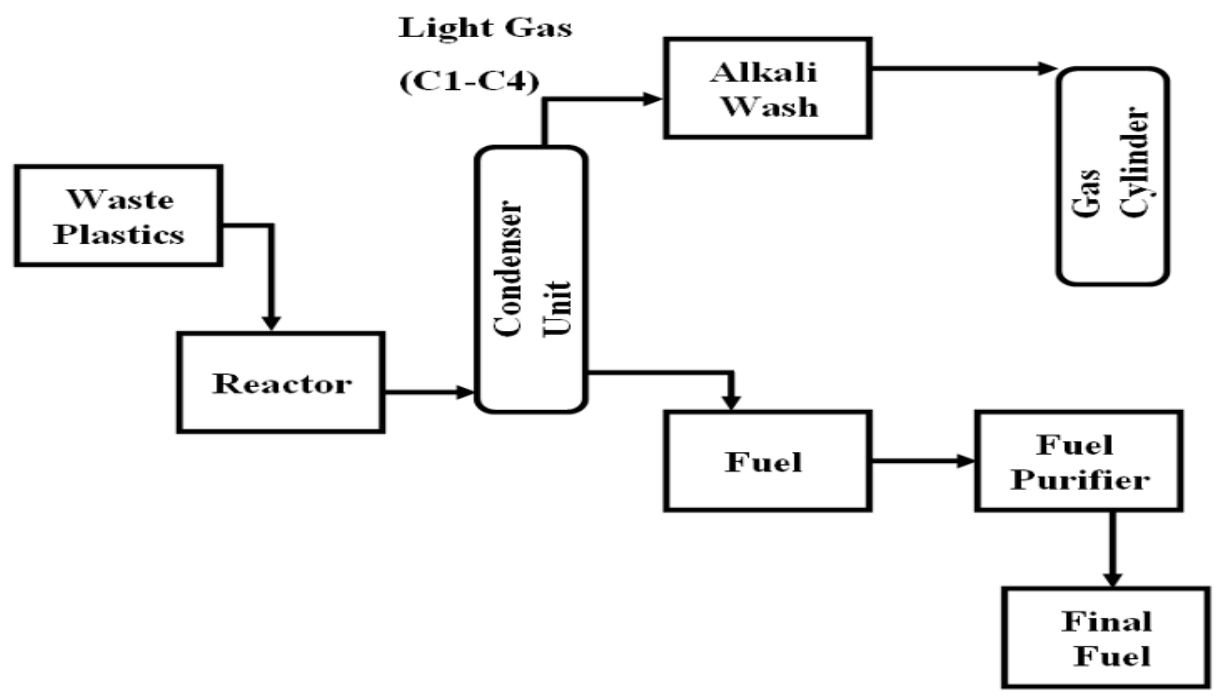

Fig. 5. Individual \& Waste Plastic to Fuel Production Process Diagram

(LDPE, code 4), polypropylene (PP, code 5) and polystyrene (PS, code 6). These processes were investigated with mixture of several plastics such as HDPE-2, LDPE-4, and PP-5 \&PS6. These waste plastics are collected, optionally sorted, cleaned of contaminants, and shredded into small pieces prior to the thermal degradation process. The experiment could be randomly mixture of waste plastics or proportional ratio mixture of waste plastics. For small-scale laboratory process the weight of input waste plastics ranges from 300 grams to $3 \mathrm{~kg}$. In the laboratory processes our present reactor chamber capacity is $2-3 \mathrm{~kg}$. We put $2 \mathrm{~kg}$ of grinding sample into the reactor chamber to expedite the experiment process. At the starting point of experiment reactor temperature set up at $350{ }^{\circ} \mathrm{C}$ for quick melting, after melted temperature maintained manually from "reactor temperature profile menu option" by increasing and decreasing depending to the rate of reaction. The optimum temperature (steady \& more fuel production state) is $305^{\circ} \mathrm{C}$. From $2 \mathrm{~kg}$ of waste plastics obtained fuel amount is 2 liter $600 \mathrm{ml}(2600 \mathrm{ml})$, fuel density is $0.76 \mathrm{~g} / \mathrm{ml}$. We defined the fuel as heating oil named "NSR fuel". The experiment additionally produced light gases Methane, Ethane, Propane and Butane as well as few amount of carbon ashes as a remaining residue. These light gases would be the alternative source of natural gases. Mixed waste plastic to produced fuel preliminary test indicated that the hydrocarbon compound rage from $C_{3}$ to $\mathrm{C}_{27}$.

\subsection{Fractional distillation process}

Fractional distillation process has been conducted according to the laboratory scale. We measured $700 \mathrm{ml}$ of NSR fuel called heating fuel and took the weight of $1000 \mathrm{ml}$ boiling flask (Glass Reactor). Subsequently fuel poured into the boiling flask, after that we put filled boiling flask in $1000 \mathrm{ml}$ heat mantle as well as connected variac meter with heat mantle. Attached distillation adapter, clump joint, condenser and collection flask with high temperature apiezon grease and insulated by aluminum foil paper. Initially we ran the experiment at $40^{\circ} \mathrm{C}$ to collect gasoline grade, after gasoline collection subsequently we raised the temperature to $110^{\circ} \mathrm{C}$ for 
naphtha (Chemical), $180{ }^{\circ} \mathrm{C}$ for aviation fuel, $260^{\circ} \mathrm{C}$ for diesel fuel and eventually at $340{ }^{\circ} \mathrm{C}$ we found fuel oil. At the end of the experiment remaining residual fuel was less, approximately amount 10-15 ml. Out of $700 \mathrm{ml}$ NSR fuel we collected $125 \mathrm{ml}$ of gasoline; density is $0.72 \mathrm{~g} / \mathrm{ml}$, $150 \mathrm{ml}$ of naphtha; density is $0.73,200 \mathrm{ml}$ of aviation fuel; density is $0.74,150 \mathrm{ml}$ of diesel fuel; density is $0.80 \mathrm{~g} / \mathrm{ml}$ and $50-60 \mathrm{ml}$ of fuel oil; density is 0.84 .

\section{Fuel production yield percentage}

After all experiment done on behalf of each experiment we calculated the yield percentages of fuel production, light gases and residue. In addition described the physical properties of each fuel such as fuel density, specific gravity, fuel color and fuel appearance respectively. Similarly, individual fuel production yield percentages \& properties are given below in Table 8 (a) \& 9 (a) and Mixed Waste Plastics to fuel Yield percentages \& properties are also given below in Table $8(\mathrm{~b}) \& 9$ (b).

\begin{tabular}{cccc}
\hline Waste Plastic Name & Fuel Yield \% & Light Gas \% & Residue \% \\
\hline HDPE-2 & 89.354 & 5.345 & 5.299 \\
LDPE-4 & 87.972 & 5.806 & 6.221 \\
PP-5 & 91.981 & 2.073 & 5.944 \\
PS-6 & 85.331 & 4.995 & 9.674 \\
\hline
\end{tabular}

Table 8. (a): Individual Fuel Production Yield Percentage

\begin{tabular}{cccc}
\hline Sample Name & Fuel Yield $\%$ & Light Gas $\%$ & Residue $\%$ \\
\hline HDPE,LDPE,PP\&PS & 90 & 5 & 5 \\
\hline
\end{tabular}

Table 8. (b): Mixed Waste Plastic to Fuel Yield Percentage

\begin{tabular}{|c|c|c|c|c|}
\hline $\begin{array}{c}\text { Name of Waste } \\
\text { Plastic Fuel }\end{array}$ & $\begin{array}{c}\text { Fuel Density } \\
\mathrm{gm} / \mathrm{ml}\end{array}$ & $\begin{array}{l}\text { Specific } \\
\text { Gravity }\end{array}$ & Fuel Color & Fuel Appearance \\
\hline LDPE-4 & 0.771 & 0.7702 & $\begin{array}{l}\text { Yellow, light } \\
\text { transparent }\end{array}$ & $\begin{array}{l}\text { Little bit wax and } \\
\text { ash content }\end{array}$ \\
\hline HDPE-2 & 0.782 & 0.7812 & $\begin{array}{l}\text { Yellow, no } \\
\text { transparent }\end{array}$ & $\begin{array}{l}\text { Wax, cloudy and } \\
\text { little bit ash content }\end{array}$ \\
\hline PP-5 & 0.759 & 0.7582 & $\begin{array}{l}\text { Light brown, } \\
\text { light } \\
\text { transparent }\end{array}$ & $\begin{array}{l}\text { Little bit wax and } \\
\text { ash content }\end{array}$ \\
\hline PS-6 & 0.916 & 0.9150 & $\begin{array}{l}\text { Light yellow, } \\
\text { not transparent }\end{array}$ & $\begin{array}{l}\text { Wax, cloudy and } \\
\text { little bit ash content }\end{array}$ \\
\hline
\end{tabular}

Table 9. (a): Individual Plastic to Fuel Properties

\begin{tabular}{ccccc}
\hline Name of Fuel & Density $\mathbf{g} / \mathbf{m l}$ & $\begin{array}{c}\text { Specific } \\
\text { Gravity }\end{array}$ & Fuel Color & Fuel \\
\hline $\begin{array}{c}\text { Mixed Plastic } \\
\text { to Fuel }\end{array}$ & 0.775 & 0.7742 & $\begin{array}{c}\text { Yellow light } \\
\text { transparent }\end{array}$ & $\begin{array}{c}\text { Ash contain } \\
\text { present }\end{array}$ \\
\hline
\end{tabular}

Table 9. (b): Mixed Waste Plastic to Fuel Properties 


\subsection{Fuel analysis and result discussion}

4.2 Gas Chromatography and Mass Spectrometer (GC/MS) analysis

Analysis of Individual waste plastics (HDPE-2, LDPE-4, PP-5, and PS-6) to individual fuel:

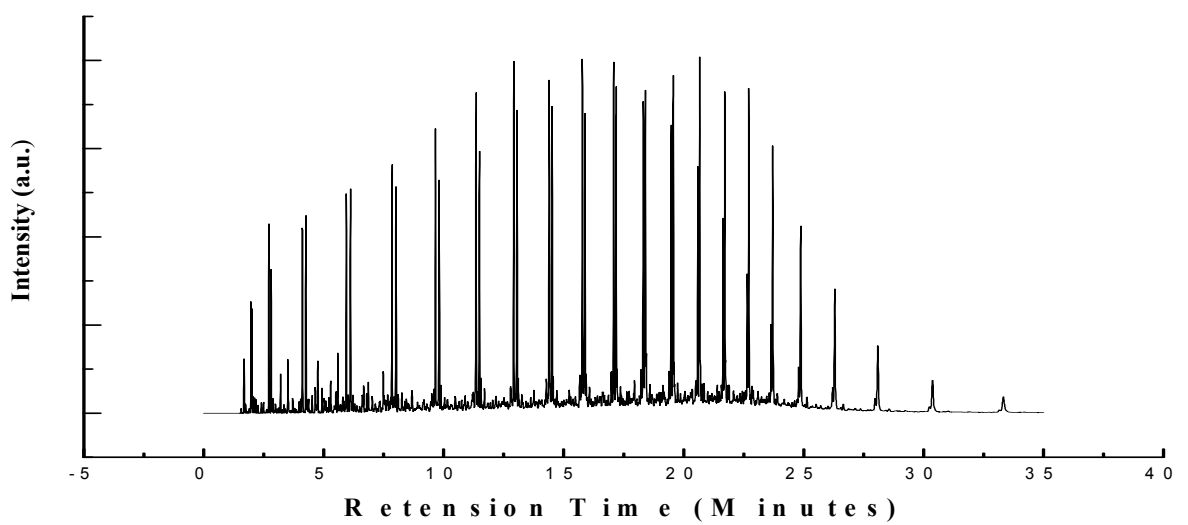

Fig. 6. GC/MS Chromatogram of HDPE-2 Waste Plastic to Fuel

\begin{tabular}{|c|c|c|c|c|c|}
\hline $\begin{array}{l}\text { Retention } \\
\text { Time } \\
\text { (Minutes) }\end{array}$ & $\begin{array}{l}\text { Compound } \\
\text { Name }\end{array}$ & Formula & $\begin{array}{l}\text { Retention } \\
\text { Time } \\
\text { (Minutes) }\end{array}$ & $\begin{array}{l}\text { Compound } \\
\text { Name }\end{array}$ & Formula \\
\hline 1.56 & Propane & $\mathrm{C}_{3} \mathrm{H}_{8}$ & 12.18 & $\begin{array}{l}\text { Cyclopentane, } \\
\text { hexyl- }\end{array}$ & $\mathrm{C}_{11} \mathrm{H}_{22}$ \\
\hline 1.66 & 2-Butene, (E)- & $\mathrm{C}_{4} \mathrm{H}_{8}$ & 12.92 & 1-Dodecene & $\mathrm{C}_{12} \mathrm{H}_{24}$ \\
\hline 1.68 & Butane & $\mathrm{C}_{4} \mathrm{H}_{10}$ & 13.05 & Dodecane & $\mathrm{C}_{12} \mathrm{H}_{26}$ \\
\hline 1.96 & $\begin{array}{c}\text { Cyclopropane, } \\
\text { 1,2-dimethyl-, } \\
\text { cis- }\end{array}$ & $\mathrm{C}_{5} \mathrm{H}_{10}$ & 13.76 & Cyclododecane & $\mathrm{C}_{12} \mathrm{H}_{24}$ \\
\hline 9.65 & 1-Decene & $\mathrm{C}_{10} \mathrm{H}_{20}$ & 27.98 & 1-Docosene & $\mathrm{C}_{22} \mathrm{H}_{44}$ \\
\hline 9.80 & Decane & $\mathrm{C}_{10} \mathrm{H}_{22}$ & 28.09 & Tetracosane & $\mathrm{C}_{24} \mathrm{H}_{50}$ \\
\hline 11.35 & 1-Undecene & $\mathrm{C}_{11} \mathrm{H}_{22}$ & 30.24 & 1-Docosene & $\mathrm{C}_{22} \mathrm{H}_{44}$ \\
\hline 11.49 & Undecane & $\mathrm{C}_{11} \mathrm{H}_{24}$ & 30.38 & Octacosane & $\mathrm{C}_{28} \mathrm{H}_{58}$ \\
\hline
\end{tabular}

Table 10. GC/MS Chromatogram Compound List of HDPE-2 Waste Plastic to Fuel 


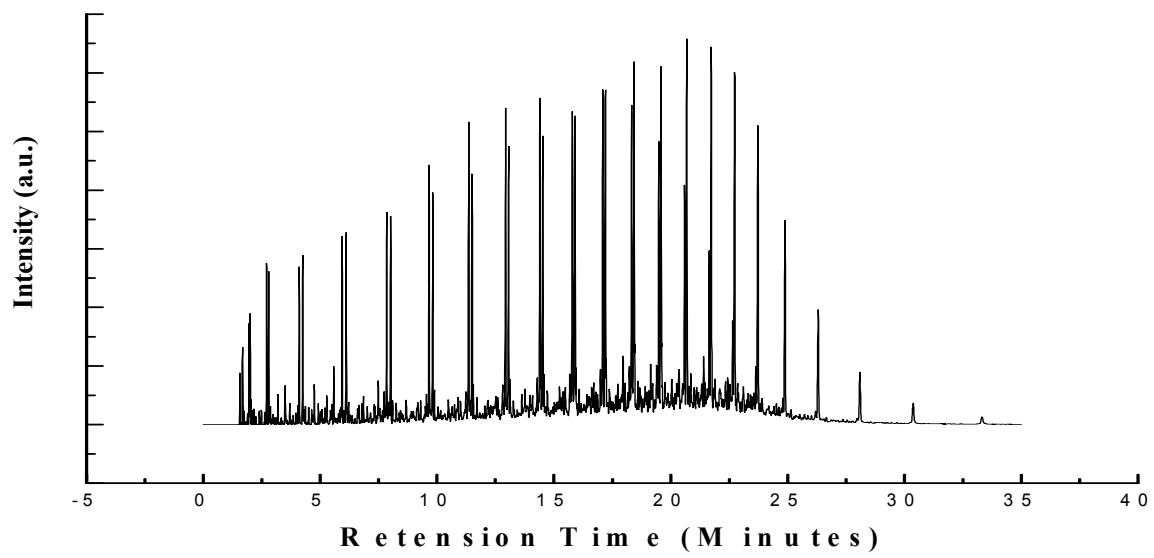

Fig. 7. GC/MS Chromatogram of LDPE-4 Waste Plastic to Fuel

\begin{tabular}{cccccc}
\hline $\begin{array}{c}\text { Retention } \\
\text { Time } \\
\text { ( Minutes) }\end{array}$ & $\begin{array}{c}\text { Compound } \\
\text { Name }\end{array}$ & $\begin{array}{c}\text { Compound } \\
\text { Formula }\end{array}$ & $\begin{array}{c}\text { Retention } \\
\text { Time } \\
\text { (Minutes) }\end{array}$ & $\begin{array}{c}\text { Compound } \\
\text { Name }\end{array}$ & $\begin{array}{c}\text { Compound } \\
\text { Formula }\end{array}$ \\
\hline 1.55 & Cyclopropane & $\mathrm{C}_{3} \mathrm{H}_{6}$ & 12.92 & 1-Dodecene & $\mathrm{C}_{12} \mathrm{H}_{24}$ \\
1.68 & Butane & $\mathrm{C}_{4} \mathrm{H}_{10}$ & 13.06 & Dodecane & $\mathrm{C}_{12} \mathrm{H}_{26}$ \\
1.96 & 2-Pentene, (E)- & $\mathrm{C}_{5} \mathrm{H}_{10}$ & 13.76 & Cyclododecane & $\mathrm{C}_{12} \mathrm{H}_{24}$ \\
1.99 & Pentane & $\mathrm{C}_{5} \mathrm{H}_{12}$ & 14.40 & 1-Tridecene & $\mathrm{C}_{13} \mathrm{H}_{26}$ \\
10.48 & Cyclodecane & $\mathrm{C}_{10} \mathrm{H}_{20}$ & 24.88 & Heneicosane & $\mathrm{C}_{21} \mathrm{H}_{44}$ \\
10.89 & Cyclohexene, 3- & & & Heneicosane & $\mathrm{C}_{21} \mathrm{H}_{44}$ \\
11.35 & $\begin{array}{c}\text { (2- } \\
11.49\end{array}$ & $\mathrm{C}_{10} \mathrm{H}_{18}$ & 26.31 & Tetracosane & $\mathrm{C}_{24} \mathrm{H}_{50}$ \\
\hline
\end{tabular}

Table 11. GC/MS Chromatogram Compound List of LDPE-4 Waste Plastic to Fuel 


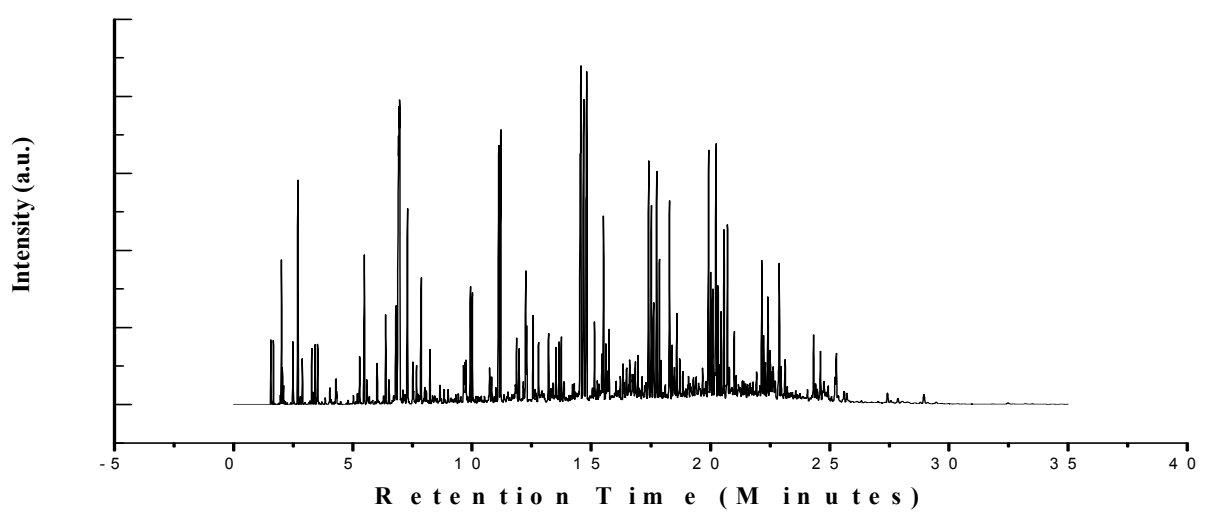

Fig. 8. GC/MS Chromatogram of PP-5 Waste Plastic to Fuel

\begin{tabular}{|c|c|c|c|c|c|}
\hline $\begin{array}{c}\text { Retention } \\
\text { Time } \\
\text { (Minute) }\end{array}$ & $\begin{array}{l}\text { Compound } \\
\text { Name }\end{array}$ & Formula & $\begin{array}{l}\text { Retention } \\
\text { Time } \\
\text { (Minute) }\end{array}$ & Compound Name & Formula \\
\hline 1.55 & Cyclopropane & $\mathrm{C}_{3} \mathrm{H}_{6}$ & 11.13 & $\begin{array}{l}\text { Cyclooctane, 1,4- } \\
\text { dimethyl-, cis- }\end{array}$ & $\mathrm{C}_{10} \mathrm{H}_{20}$ \\
\hline 1.66 & $\begin{array}{l}\text { 1-Propene, 2- } \\
\text { methyl- }\end{array}$ & $\mathrm{C}_{4} \mathrm{H}_{8}$ & 11.20 & 1-Tetradecene & $\mathrm{C}_{14} \mathrm{H}_{28}$ \\
\hline 1.99 & Pentane & $\mathrm{C}_{5} \mathrm{H}_{12}$ & 11.86 & $\begin{array}{l}\text { 1-Dodecanol, 3,7,11- } \\
\text { trimethyl- }\end{array}$ & $\mathrm{C}_{15} \mathrm{H}_{32} \mathrm{O}$ \\
\hline 2.48 & $\begin{array}{l}\text { Pentane, 2- } \\
\text { methyl- }\end{array}$ & $\mathrm{C}_{6} \mathrm{H}_{14}$ & 12.25 & $\begin{array}{c}(2,4,6- \\
\text { Trimethylcyclohexyl) } \\
\text { methanol }\end{array}$ & $\mathrm{C}_{10} \mathrm{H}_{20} \mathrm{O}$ \\
\hline 9.64 & $\begin{array}{l}\text { Nonane, 2- } \\
\text { methyl-3- } \\
\text { methylene- }\end{array}$ & $\mathrm{C}_{11} \mathrm{H}_{22}$ & 23.13 & $\begin{array}{c}\text { Dodecane, } 1- \\
\text { cyclopentyl-4-(3- } \\
\text { cyclopentylypropyl)- }\end{array}$ & $\mathrm{C}_{25} \mathrm{H}_{48}$ \\
\hline 9.74 & $\begin{array}{l}\text { 3-Undecene, } \\
\text { (Z)- }\end{array}$ & $\mathrm{C}_{11} \mathrm{H}_{22}$ & 25.72 & $\begin{array}{l}\text { Cyclotetradecane } \\
\text { 1,7,11-trimethyl-4-(1- } \\
\text { methylethyl)- }\end{array}$ & $\mathrm{C}_{20} \mathrm{H}_{40}$ \\
\hline 9.92 & $\begin{array}{c}\text { Octane, 3,3- } \\
\text { dimethyl- }\end{array}$ & $\mathrm{C}_{10} \mathrm{H}_{22}$ & 28.95 & $\begin{array}{c}\text { Dodecane, 1- } \\
\text { cyclopentyl-4-(3- } \\
\text { cyclopentylypropyl)- }\end{array}$ & $\mathrm{C}_{25} \mathrm{H}_{48}$ \\
\hline 10.73 & $\begin{array}{l}\text { 3-Decene, 2,2- } \\
\text { dimethyl-, } \\
\text { (E)- }\end{array}$ & $\mathrm{C}_{12} \mathrm{H}_{24}$ & & & \\
\hline
\end{tabular}

Table 12. GC/MS Chromatogram Compound List of PP-5 Waste Plastic to Fuel 


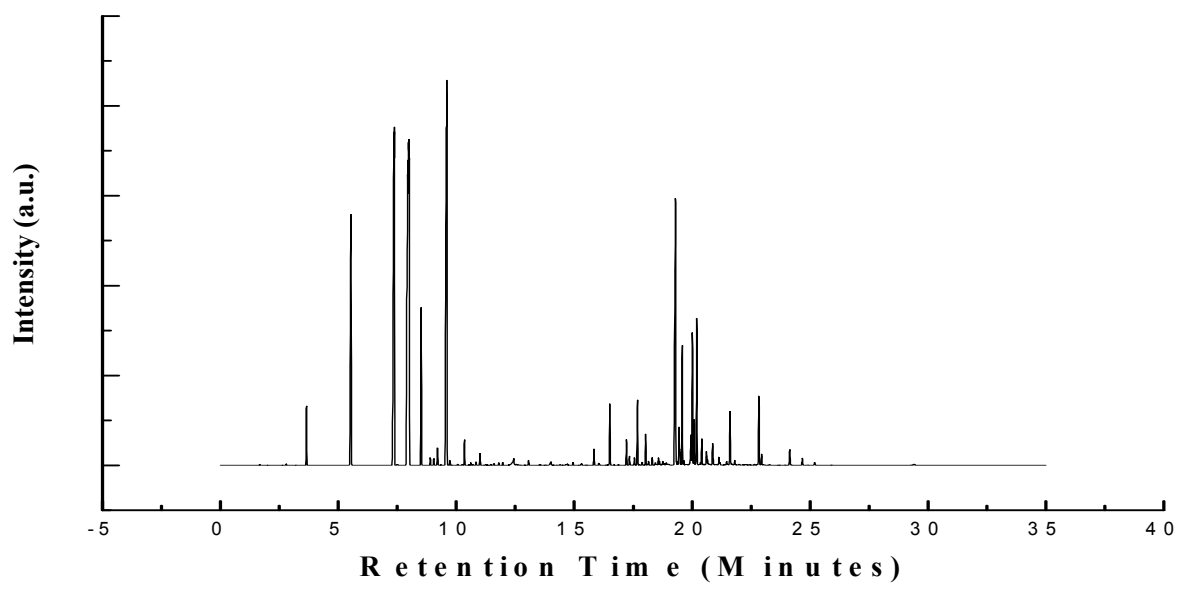

Fig. 9. GC/MS Chromatogram of PS-6 Waste Plastic to Fuel

\begin{tabular}{|c|c|c|c|c|c|}
\hline $\begin{array}{l}\text { Retention } \\
\text { Time } \\
\text { (Minute) }\end{array}$ & $\begin{array}{l}\text { Compound } \\
\text { Name }\end{array}$ & Formula & $\begin{array}{l}\text { Retention } \\
\text { Time } \\
\text { (Minute) }\end{array}$ & Compound Name & Formula \\
\hline 3.65 & 1,5-Hexadiyne & $\mathrm{C}_{6} \mathrm{H}_{6}$ & 17.68 & $\begin{array}{l}\text { Benzene, 1,1'-(1,2- } \\
\text { ethanediyl)bis- }\end{array}$ & $\mathrm{C}_{14} \mathrm{H}_{14}$ \\
\hline 5.54 & Toluene & $\mathrm{C}_{7} \mathrm{H}_{8}$ & 18.03 & $\begin{array}{c}\text { Benzene, 1,1'-(1- } \\
\text { methyl-1,2- } \\
\text { ethanediyl)bis- }\end{array}$ & $\mathrm{C}_{15} \mathrm{H}_{16}$ \\
\hline 7.94 & Styrene & $\mathrm{C}_{8} \mathrm{H}_{8}$ & 19.30 & $\begin{array}{l}\text { Benzene, } 1,1^{\prime}-(1,3- \\
\text { propanediyl)bis- }\end{array}$ & $\mathrm{C}_{15} \mathrm{H}_{16}$ \\
\hline 11.00 & Acetophenone & $\mathrm{C}_{8} \mathrm{H}_{8} \mathrm{O}$ & 21.61 & $\begin{array}{l}\text { Naphthalene,1- } \\
\text { phenyl- }\end{array}$ & $\mathrm{C}_{16} \mathrm{H}_{12}$ \\
\hline 13.07 & Naphthalene & $\mathrm{C}_{10} \mathrm{H}_{8}$ & 21.81 & o-Terphenyl & $\mathrm{C}_{18} \mathrm{H}_{14}$ \\
\hline 15.84 & Biphenyl & $\mathrm{C}_{12} \mathrm{H}_{10}$ & 22.83 & $\frac{2-}{\text { Phenylnaphthalene }}$ & $\mathrm{C}_{16} \mathrm{H}_{12}$ \\
\hline 16.51 & Diphenylmethane & $\mathrm{C}_{13} \mathrm{H}_{12}$ & 24.14 & $\begin{array}{l}\text { 9-Phenyl-5H- } \\
\text { benzocycloheptene }\end{array}$ & $\mathrm{C}_{17} \mathrm{H}_{14}$ \\
\hline 17.22 & $\begin{array}{l}\text { Benzene,1,1'- } \\
\text { ethylidenebis- }\end{array}$ & $\mathrm{C}_{14} \mathrm{H}_{14}$ & 24.67 & p-Terphenyl & $\mathrm{C}_{18} \mathrm{H}_{14}$ \\
\hline
\end{tabular}

Table 13. GC/MS Chromatogram Compound List of PS-6 Waste Plastic to Fuel

From GCMS analysis of Individual HDPE-2, LDPE-4, PP-5, and PS-6 fuel, in accordance with their numerous retention times many compounds are found, some of them are mentioned shortly. In HDPE-2 fuel at retention time 1.56, compound is Propane $\left(\mathrm{C}_{3} \mathrm{H}_{8}\right)$, and finally at retention time 30.38 obtained compound is Octacosane $\left(\mathrm{C}_{28} \mathrm{H}_{58}\right)$, [Shown above, Fig. 6 \& Table-10]. In LDPE-4 fuel at retention time 1.55, compound is Cyclopropane $\left(\mathrm{C}_{3} \mathrm{H}_{6}\right)$, and finally at retention time 33.21 obtained compound is Octacosane $\left(\mathrm{C}_{28} \mathrm{H}_{58}\right)$ [Shown above, 
Fig.7 \& Table-11]. In PP-5 initially at retention time 1.55 compound is Cyclopropane $\left(\mathrm{C}_{3} \mathrm{H}_{6}\right)$ and finally at retention time 28.95 obtained compound is Dodecane,-1-Cyclopentyl-4-(3Cyclopentylpropyl) $\left(\mathrm{C}_{22} \mathrm{H}_{46} \mathrm{O}_{2}\right)$ [Shown above, Fig.8 \& Table-12] . Accordingly in PS-6 at retention time 3.65 found compound is 1,5-Hexadiyne and eventually at retention time 24.67 obtained compound is p-Terphnyl $\left(\mathrm{C}_{18} \mathrm{H}_{14}\right)$ [Shown above, Fig.9 \& Table-13].

\section{Analysis of Mixed Waste Plastics to Fuel (Heating Oil):}

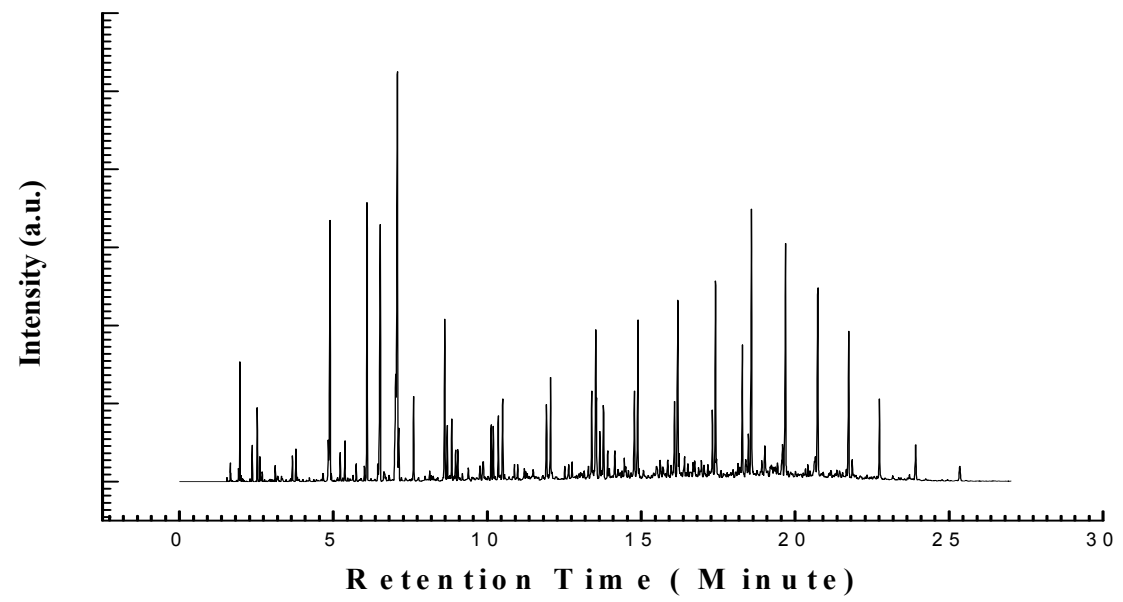

Fig. 10. GC/MS Chromatogram of Mixed Waste Plastic to Fuel (Heating Oil)

\begin{tabular}{cccc}
\hline Compound Name & Formula & Compound Name & Formula \\
\hline Cyclopropane & $(\mathrm{C} 3 \mathrm{H} 6)$ & $\begin{array}{c}\text { Dodecane } \\
\text { Decane, 2,3,5,8- } \\
\text { tetramethyl- }\end{array}$ & $(\mathrm{C} 12 \mathrm{H} 26)$ \\
2-Butene, $(\mathrm{E})-$ & $(\mathrm{C} 4 \mathrm{H} 8)$ & $\begin{array}{c}\text { 1-Tridecene } \\
\text { Tridecane }\end{array}$ & $(\mathrm{C} 14 \mathrm{H} 30)$ \\
$\begin{array}{c}\text { Pentane } \\
\text { Cycloproper 2-methyl- }\end{array}$ & $(\mathrm{C} 5 \mathrm{H} 12)$ & Heneicosane & $(\mathrm{C} 13 \mathrm{H} 28)$ \\
$\begin{array}{c}\text { heptyl-2-methyl- } \\
\text { Undecane }\end{array}$ & $(\mathrm{C} 11 \mathrm{H} 22)$ & Nonadecane & $(\mathrm{C} 21 \mathrm{H} 44)$ \\
$\begin{array}{c}\text { 1-Dodecanol, 3,7,11- } \\
\text { trimethyl- }\end{array}$ & $(\mathrm{C} 11 \mathrm{H} 24)$ & Benzene, hexadecyl- & $(\mathrm{C} 19 \mathrm{H} 40)$ \\
1-Dodecene & $(\mathrm{C} 15 \mathrm{H} 32 \mathrm{O})$ & Heptacosane & $(\mathrm{C} 22 \mathrm{H} 38)$ \\
\hline
\end{tabular}

Table 14. GC/MS Chromatogram Compound List of Mixed Waste Plastic to Fuel (Heating Oil)

From GCMS analysis of NSR fuel (Called Heating Fuel) primarily we found long chain hydrocarbon of compound. In the GCMS data we have noticed that the obtained compounds are Cyclopropane $\left(\mathrm{C}_{3} \mathrm{H}_{6}\right)$ to Heptacosane $\left(\mathrm{C}_{27} \mathrm{H}_{56}\right)$ including long and short chain of hydrocarbon compound [Shown above, Fig.10 \& Table-14]. 


\section{GCMS Analysis of Mixed Waste Plastics to Fractional Distillation Fuel:}

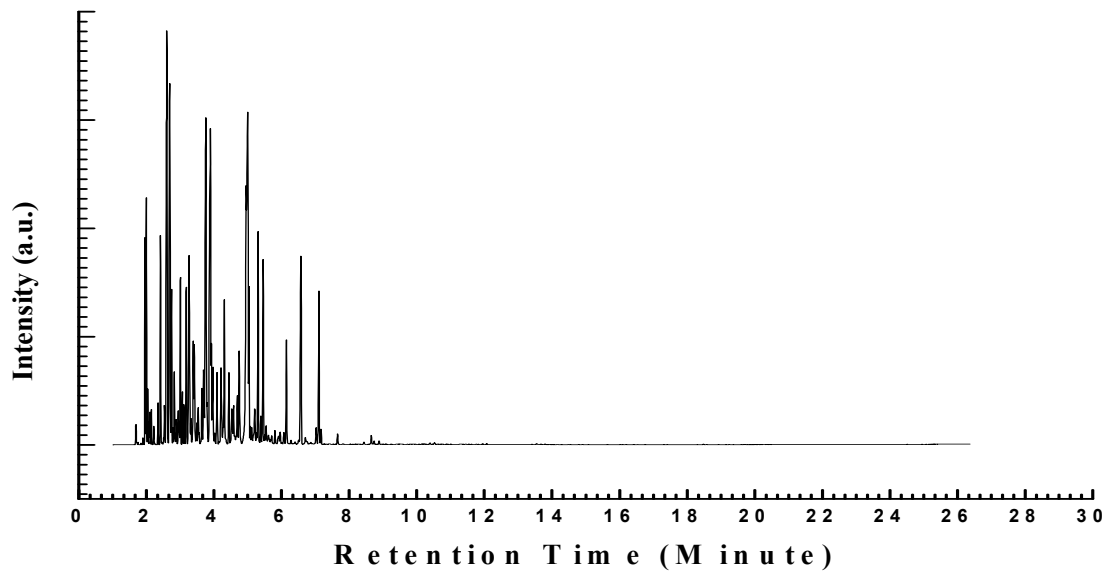

Fig. 11. GC/MS Chromatogram of Mixed Waste Plastic Fuel to 1 ${ }^{\text {st }}$ Fractional Fuel (Gasoline)

\begin{tabular}{llll}
\hline Compound Name & Formula & Compound Name & Formula \\
\hline 1-Propene,2-methyl- & $(\mathrm{C} 4 \mathrm{H} 8)$ & Heptane & $(\mathrm{C} 7 \mathrm{H} 16)$ \\
Butane & $(\mathrm{C} 4 \mathrm{H} 10)$ & 1,4-hexadiene,4-methyl- & $(\mathrm{C} 7 \mathrm{H} 12)$ \\
2-Pentene & $(\mathrm{C} 5 \mathrm{H} 10)$ & 1,4-Heptadiene & $(\mathrm{C} 7 \mathrm{H} 12)$ \\
2-Pentene, $(\mathrm{E})$ & $(\mathrm{C} 5 \mathrm{H} 10)$ & Cyclohexane,methyl- & $(\mathrm{C} 7 \mathrm{H} 14)$ \\
Cyclohexane & $(\mathrm{C} 6 \mathrm{H} 12)$ & 1-Nonane & $(\mathrm{C} 9 \mathrm{H} 18)$ \\
Hexane,3-methyl & $(\mathrm{C} 7 \mathrm{H} 16)$ & Styrene & $(\mathrm{C} 8 \mathrm{H} 8)$ \\
Cyclohexene & $(\mathrm{C} 6 \mathrm{H} 10)$ & Nonane & $(\mathrm{C} 9 \mathrm{H} 20)$ \\
1-Hexene,2-methyl- & $(\mathrm{C} 7 \mathrm{H} 14)$ & Benzene,(1-methylethyl)- & $(\mathrm{C} 9 \mathrm{H} 12)$ \\
1-Heptane & $(\mathrm{C} 7 \mathrm{H} 14)$ & & \\
\hline
\end{tabular}

Table 15. GC/MS Chromatogram compound list of Mixed Waste Plastic Fuel to $1^{\text {st }}$ Fractional Fuel (Gasoline) 


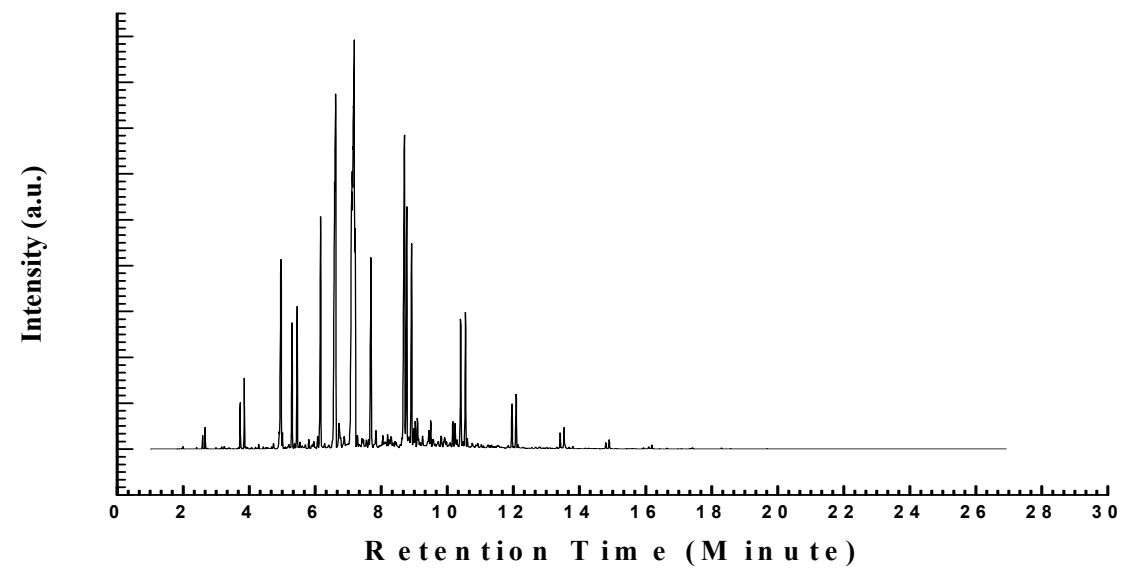

Fig. 12. GC/MS Chromatogram of Mixed Waste Plastic Fuel to 2nd Fractional Fuel (Naphtha, Chemical)

\begin{tabular}{llll}
\hline Compound Name & Formula & Compound Name & Formula \\
\hline 1-Hexene & $(\mathrm{C} 6 \mathrm{H} 12)$ & Cyclopentane-butyl- & $(\mathrm{C} 9 \mathrm{H} 8)$ \\
Hexane & $(\mathrm{C} 6 \mathrm{H} 14)$ & Benzene,propyl & $(\mathrm{C} 9 \mathrm{H} 12)$ \\
1-Heptene & $(\mathrm{C} 7 \mathrm{H} 14)$ & a-methylsyrene & $(\mathrm{C} 9 \mathrm{H} 10)$ \\
Heptane & $(\mathrm{C} 7 \mathrm{H} 16)$ & 1-Decene & $(\mathrm{C} 10 \mathrm{H} 20)$ \\
2,4-dimethyl-1- & $(\mathrm{C} 9 \mathrm{H} 18)$ & $\begin{array}{l}\text { Cyclopropane,1- } \\
\text { heptene }\end{array}$ & $(\mathrm{C} 11 \mathrm{H} 22)$ \\
Ethylbenzene & $(\mathrm{C} 8 \mathrm{H} 10)$ & Undecane & $(\mathrm{C} 11 \mathrm{H} 24)$ \\
1-Nonene & $(\mathrm{C} 9 \mathrm{H} 18)$ & 1-Dodecene & $(\mathrm{C} 12 \mathrm{H} 24)$ \\
Styrene & $(\mathrm{C} 8 \mathrm{H} 8)$ & Dodecane & $(\mathrm{C} 12 \mathrm{H} 26)$ \\
$1,3,5,7-$ & $(\mathrm{C} 8 \mathrm{H} 8)$ & Tridecane & $(\mathrm{C} 13 \mathrm{H} 28)$ \\
Cyclooctatetraene & & & \\
Nonane & $(\mathrm{C} 9 \mathrm{H} 20)$ & Tetradecdane & $(\mathrm{C} 14 \mathrm{H} 30)$ \\
\hline
\end{tabular}

Table 16. GC/MS Chromatogram Compound List of Mixed Waste Plastic Fuel to 2nd Fractional Fuel (Naphtha, Chemical) 


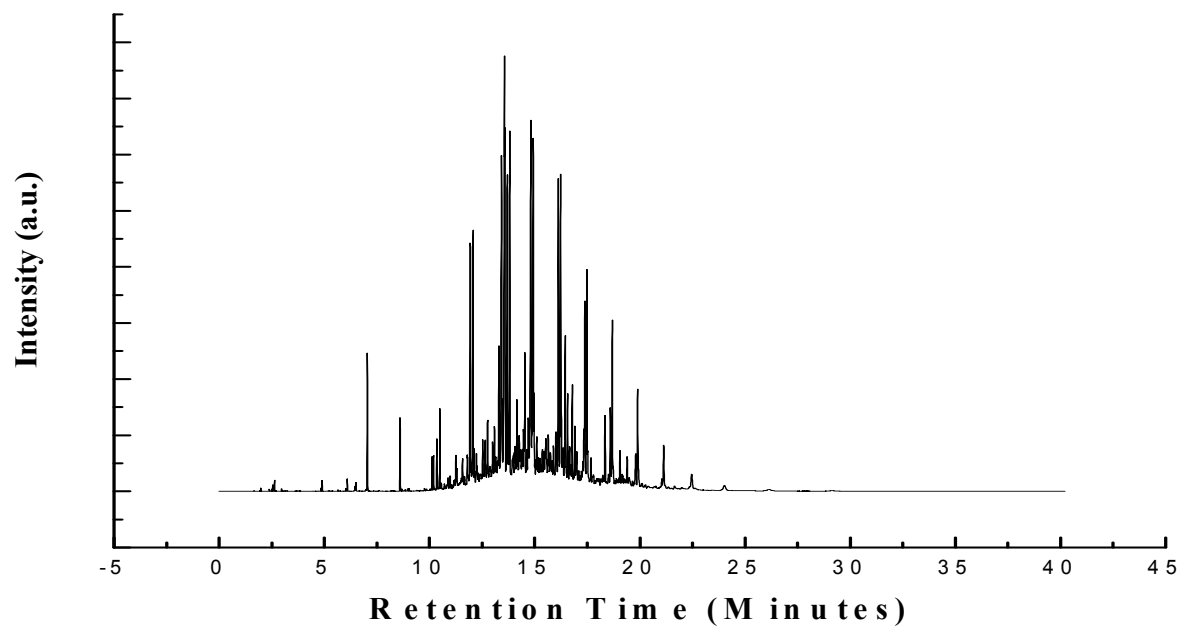

Fig. 13. GC/MS Chromatogram of Mixed Waste Plastic Fuel to $3^{\text {rd }}$ Fractional Fuel (Aviation)

\begin{tabular}{|c|c|c|c|c|c|}
\hline $\begin{array}{l}\text { Retention } \\
\text { Time } \\
\text { (Min.) }\end{array}$ & $\begin{array}{l}\text { Compound } \\
\text { Name }\end{array}$ & Formula & $\begin{array}{l}\text { Retention } \\
\text { Time } \\
\text { (Min.) }\end{array}$ & $\begin{array}{l}\text { Compound } \\
\text { Name }\end{array}$ & Formula \\
\hline 7.04 & Styrene & $\mathrm{C} 8 \mathrm{H} 8$ & 14.93 & Tetradecane & $\mathrm{C} 14 \mathrm{H} 30$ \\
\hline 8.60 & a-Methylstyrene & $\mathrm{C} 9 \mathrm{H} 10$ & 16.12 & Cyclopentadecane & C15H30 \\
\hline 10.18 & $\begin{array}{l}\text { Cyclooctane,1,4- } \\
\text { dimethyl-,cis- }\end{array}$ & $\mathrm{C} 10 \mathrm{H} 20$ & 16.23 & Pentadecane & C15H32 \\
\hline 10.38 & 1-Undecene & $\mathrm{C} 11 \mathrm{H} 22$ & 17.37 & 1-Hexadecene & C16H32 \\
\hline 12.07 & Dodecane & $\mathrm{C} 12 \mathrm{H} 26$ & 19.80 & $\begin{array}{l}\text { E-15- } \\
\text { Heptadecanal }\end{array}$ & $\mathrm{C} 17 \mathrm{H} 32 \mathrm{O}$ \\
\hline 13.42 & 1-Tridecene & $\mathrm{C} 13 \mathrm{H} 26$ & 19.89 & Octadecane & C18H38 \\
\hline 13.56 & Tridecane & C13H28 & 21.13 & Nonadecane & $\mathrm{C} 19 \mathrm{H} 40$ \\
\hline 14.81 & Cyclotetradecane & $\mathrm{C} 14 \mathrm{H} 28$ & 22.45 & Eicosane & $\mathrm{C} 2 \mathrm{OH} 42$ \\
\hline
\end{tabular}

Table 17. GC/MS Chromatogram Compound list of Mixed Waste Plastic Fuel to $3^{\text {rd }}$ Fractional Fuel (Aviation) 


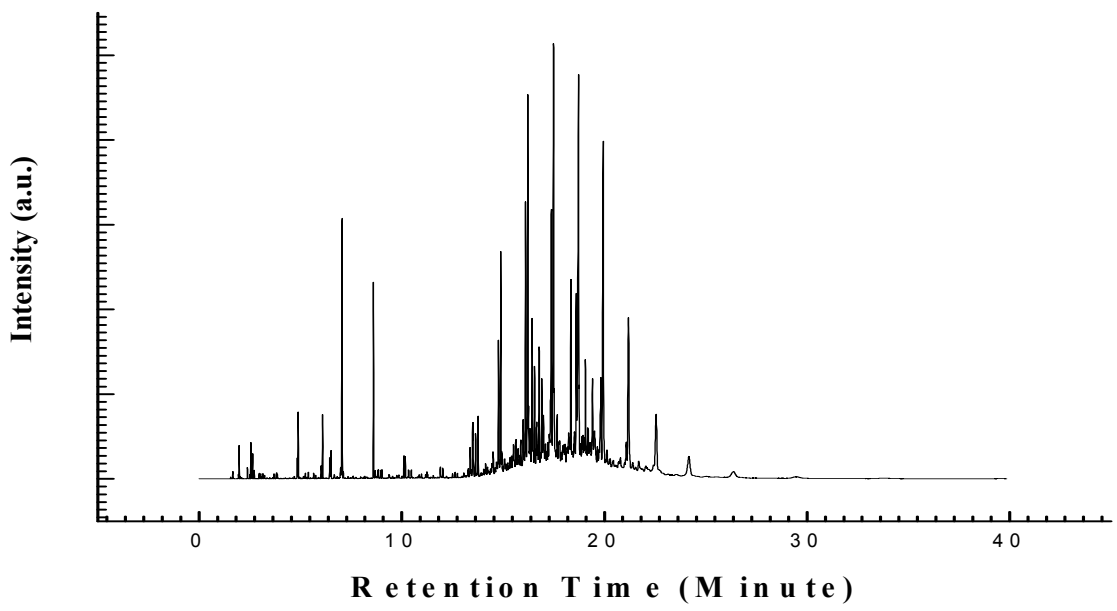

Fig. 14. GC/MS Chromatogram of Mixed Waste Plastic Fuel to $4^{\text {th }}$ Fractional Fuel (Diesel)

\begin{tabular}{llll}
\hline Compound Name & Formula & Compound Name & Formula \\
\hline Pentane & $(\mathrm{C} 5 \mathrm{H} 12)$ & 1-Pentadecene & $(\mathrm{C} 15 \mathrm{H} 30)$ \\
1-Pentene, 2-methyl- & $(\mathrm{C} 6 \mathrm{H} 12)$ & Pentadecane & $(\mathrm{C} 15 \mathrm{H} 32)$ \\
Heptane, 4-methyl- & $(\mathrm{C} 8 \mathrm{H} 18)$ & 1-Nonadecanol & $(\mathrm{C} 19 \mathrm{H} 40 \mathrm{O})$ \\
Toluene & $(\mathrm{C} 7 \mathrm{H} 8)$ & 1-Hexadecene & $(\mathrm{C} 16 \mathrm{H} 32)$ \\
E-14-Hexadecenal & $(\mathrm{C} 16 \mathrm{H} 30 \mathrm{O})$ & Eicosane & $(\mathrm{C} 20 \mathrm{H} 42)$ \\
4-Tetradecene, $(\mathrm{E})-$ & $(\mathrm{C} 14 \mathrm{H} 28)$ & Heneicosane & $(\mathrm{C} 21 \mathrm{H} 44)$ \\
Tetradecane & $(\mathrm{C} 14 \mathrm{H} 30)$ & Octacosane & $(\mathrm{C} 28 \mathrm{H} 58)$ \\
\hline
\end{tabular}

Table 18. GC/MS Chromatogram Compound List of Mixed Waste Plastic Fuel to $4^{\text {th }}$ Fractional Fuel (Diesel) 


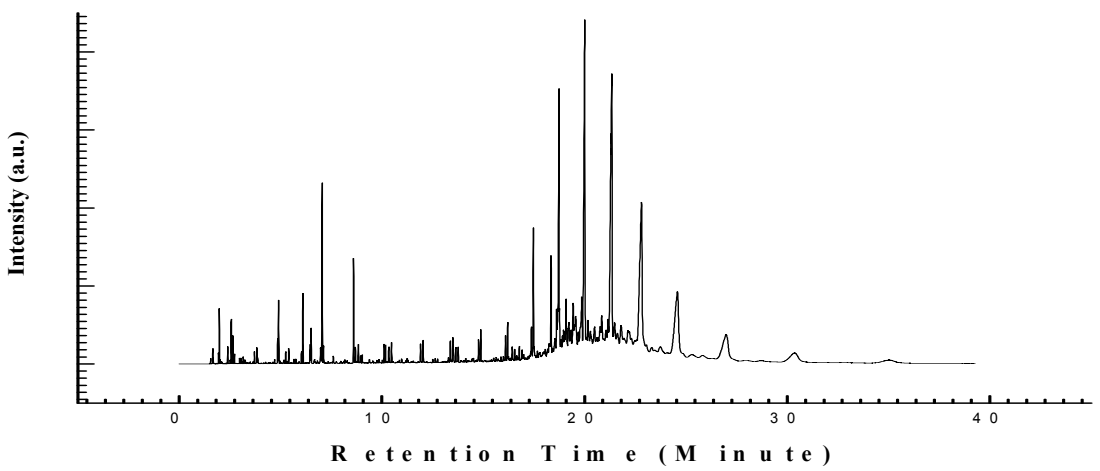

Fig. 15. GC/MS Chromatogram of Mixed Waste Plastic Fuel to $5^{\text {th }}$ Fractional Fuel (Fuel Oil)

\begin{tabular}{|c|c|c|c|}
\hline Compound Name & Formula & Compound Name & Formula \\
\hline 1) 1-Propene, 2-methyl- & (C4H8) & 16) Tridecane & $(\mathrm{C} 13 \mathrm{H} 28)$ \\
\hline 2) Pentane & (C5H12) & 17) Tetradecane & (C14H30) \\
\hline 3)1-Pentene, 2-methyl- & (C6H12) & 18) Pentadecane & (C15H32) \\
\hline 4) Hexane & (C6H14) & 19) Hexadecane & $(\mathrm{C} 16 \mathrm{H} 34)$ \\
\hline 5) Heptane & (C7H16) & $\begin{array}{l}\text { 20) Benzene, 1,1'-(1,3- } \\
\text { propanediyl)bis- }\end{array}$ & (C15H16) \\
\hline 6) à-Methylstyrene & (C9H10) & 27) Heneicosane & $(\mathrm{C} 21 \mathrm{H} 44)$ \\
\hline 7) Decane & $(\mathrm{C} 10 \mathrm{H} 22)$ & 28) Tetracosane & (C24H50) \\
\hline 8) Undecane & $(\mathrm{C} 11 \mathrm{H} 24)$ & 29) Heptacosane & (C27H56) \\
\hline
\end{tabular}

Table 19. GC/MS Chromatogram Compound list of Mixed Waste Plastic Fuel to $5^{\text {th }}$ Fractional Fuel (Fuel Oil)

GC/MS analysis of fractional distillation fuel, a lot of compound is appeared in each individual fuel. Some of those compounds are mentioned, such as in Gasoline (15T Fraction) we found Carbon range $\mathrm{C}_{4}$ to $\mathrm{C}_{9}$ and compound is 1-Propene-2-Methyl $\left(\mathrm{C}_{3} \mathrm{H}_{8}\right)$ to Benzene, (1-methylethyl) - $\left(\mathrm{C}_{9} \mathrm{H}_{12}\right)$ [Shown above, Fig.11 \& Table-15]. In naphtha (2nd Fraction) Carbon range is $\mathrm{C}_{6}$ to $\mathrm{C}_{14}$ and compound is 1- Hexene $\left(\mathrm{C}_{6} \mathrm{H}_{12}\right)$ to Tetradecane $\left(\mathrm{C}_{14} \mathrm{H}_{30}\right)$ [Shown above, Fig.12 \& Table-16]. In Aviation fuel (3rd Fraction) Carbon range is $\mathrm{C}_{8}$ to $\mathrm{C}_{20}$ and compound is Styrene $\left(\mathrm{C}_{8} \mathrm{H}_{8}\right)$ to Eicosane $\left(\mathrm{C}_{20} \mathrm{H}_{42}\right)$ [Shown above, Fig.13 \& Table-17]. In Diesel (4th Fraction) Carbon range is $\mathrm{C}_{5}$ to $\mathrm{C}_{28}$ and compound is pentane $\left(\mathrm{C}_{5} \mathrm{H}_{12}\right)$ to Octacosane $\left(\mathrm{C}_{20} \mathrm{H}_{58}\right)$ [Shown above, Fig.14 \& Table-18].Eventually in Fuel oil (5 ${ }^{\text {th }}$ Fraction) Carbon range is $\mathrm{C}_{4}$ to $\mathrm{C}_{27}$, and compound is 1-Propene-2-methyl $\left(\mathrm{C}_{4} \mathrm{H}_{8}\right)$ to Heptacosane $\left(\mathrm{C}_{27} \mathrm{H}_{56}\right)$ [Shown above, Fig.15 \& Table-19]. 


\subsection{FTIR (Spectrum-100) analysis}

Analysis of Individual waste plastics (HDPE-2, LDPE-4, PP-5, and PS-6) to individual fuel:

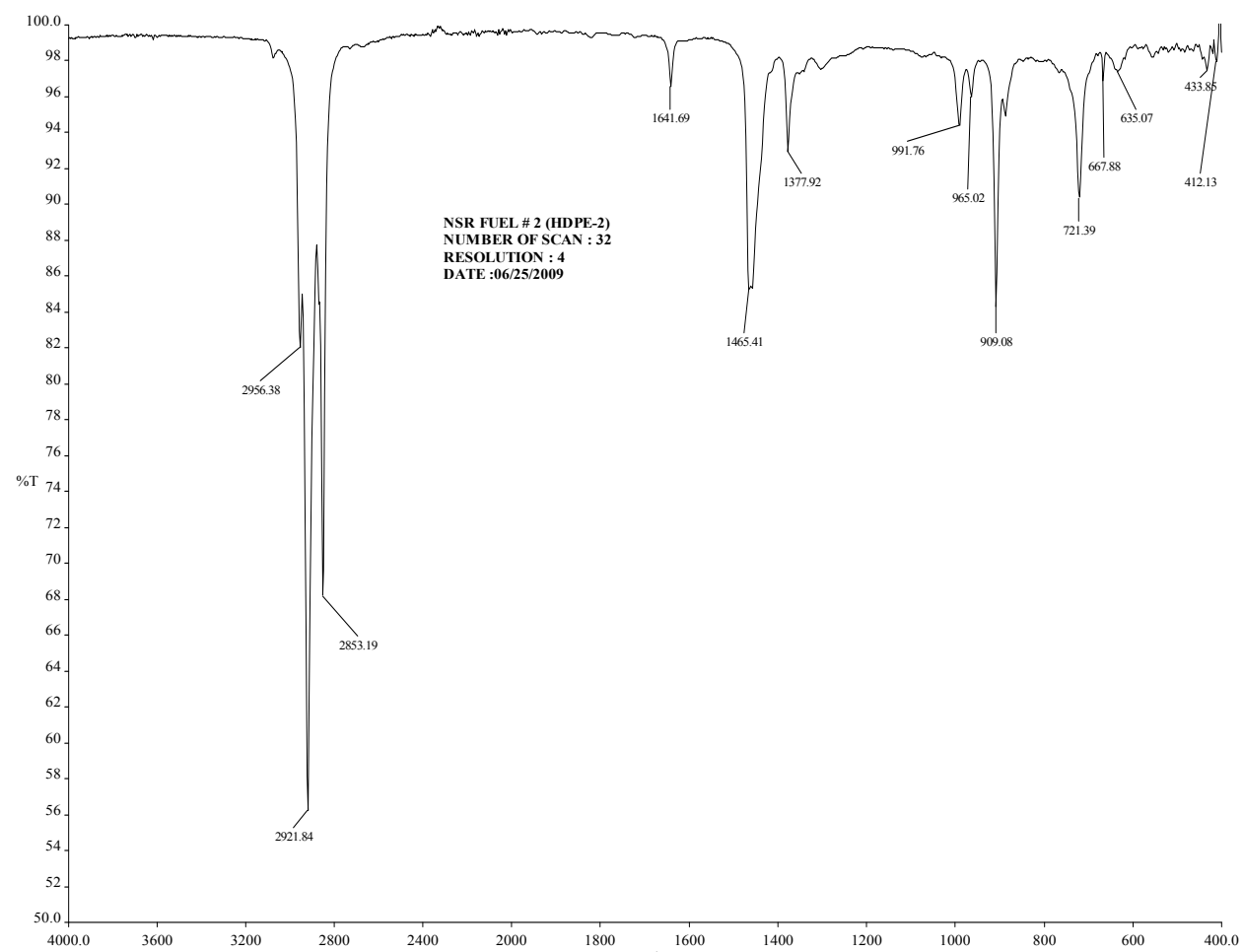

Fig. 16. FTIR Spectra of HDPE-2 Plastic to Fuel

\begin{tabular}{ccc}
\hline $\begin{array}{c}\text { Band Peak } \\
\text { Number }\end{array}$ & $\begin{array}{c}\text { Wave Number } \\
\left(\mathbf{c m}^{-1}\right)\end{array}$ & Compound Group Name \\
\hline 1 & 2956.38 & $\mathrm{C}-\mathrm{CH}_{3}$ \\
2 & 2921.84 & $\mathrm{C}-\mathrm{CH}_{3}$ \\
3 & 2853.19 & $\mathrm{CH}_{2}$ \\
4 & 1641.69 & $\mathrm{Non}-\mathrm{Conjugated}$ \\
5 & 1465.41 & $\mathrm{CH}_{3}$ \\
6 & 1377.92 & $\mathrm{CH}_{3}$ \\
7 & 991.76 & $-\mathrm{CH}=\mathrm{CH}_{2}$ \\
8 & 965.02 & $-\mathrm{CH}=\mathrm{CH}-($ Trans $)$ \\
9 & 909.08 & $-\mathrm{CH}=\mathrm{CH}_{2}$ \\
10 & 721.39 & $-\mathrm{CH}=\mathrm{CH}-(\mathrm{Cis})$ \\
11 & 667.88 & $-\mathrm{CH}=\mathrm{CH}-(\mathrm{Cis})$ \\
\hline
\end{tabular}

Table 20. FTIR Spectra of HDPE-2 Plastic to Fuel Functional Group Name 


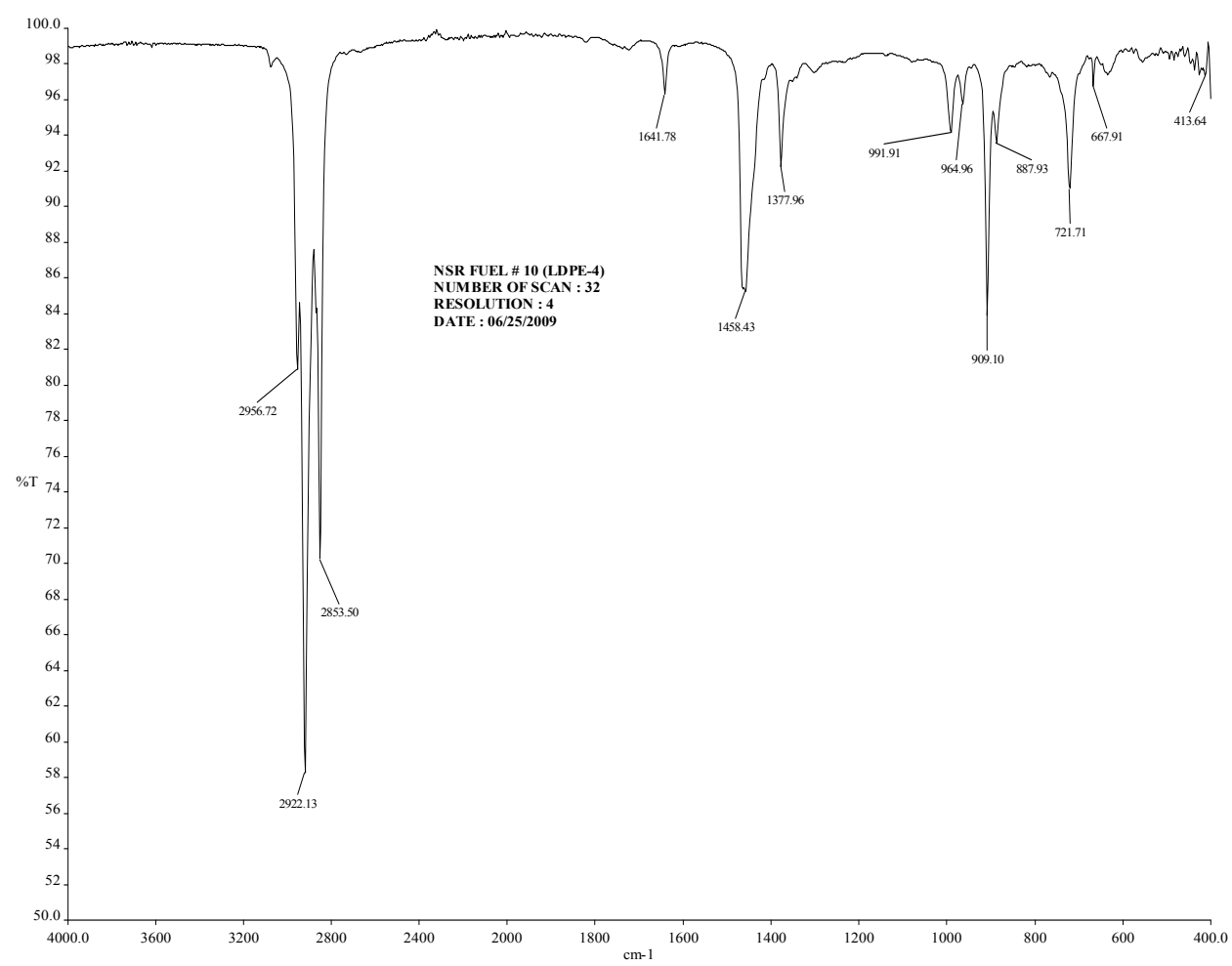

Fig. 17. FTIR Spectra of LDPE-4 Plastic to Fuel

\begin{tabular}{ccc}
\hline Band Peak Number & $\begin{array}{c}\text { Wave Number } \\
\left(\mathbf{c m}^{-1}\right)\end{array}$ & Functional Group Name \\
\hline 1 & 2956.72 & $\mathrm{C}-\mathrm{CH}_{3}$ \\
2 & 2922.13 & $\mathrm{C}-\mathrm{CH}_{3}$ \\
3 & 2853.50 & $\mathrm{CH}_{2}$ \\
4 & 1641.78 & $\mathrm{Non}-\mathrm{Conjugated}_{3}$ \\
5 & 1458.43 & $\mathrm{CH}_{3}$ \\
6 & 1377.96 & $\mathrm{CH}_{3}$ \\
7 & 964.96 & $-\mathrm{CH}=\mathrm{CH}_{2}$ \\
8 & 909.10 & $-\mathrm{CH}=\mathrm{CH}-($ Trans $)$ \\
9 & 887.93 & $-\mathrm{CH}=\mathrm{CH}_{2}$ \\
10 & 721.71 & $-\mathrm{CH}=\mathrm{CH}-(\mathrm{Cis})$ \\
11 & 667.91 & $-\mathrm{CH}=\mathrm{CH}-(\mathrm{Cis})$ \\
\hline
\end{tabular}

Table 21. FTIR Spectra of LDPE-4 Plastic to Fuel Functional Group Name 


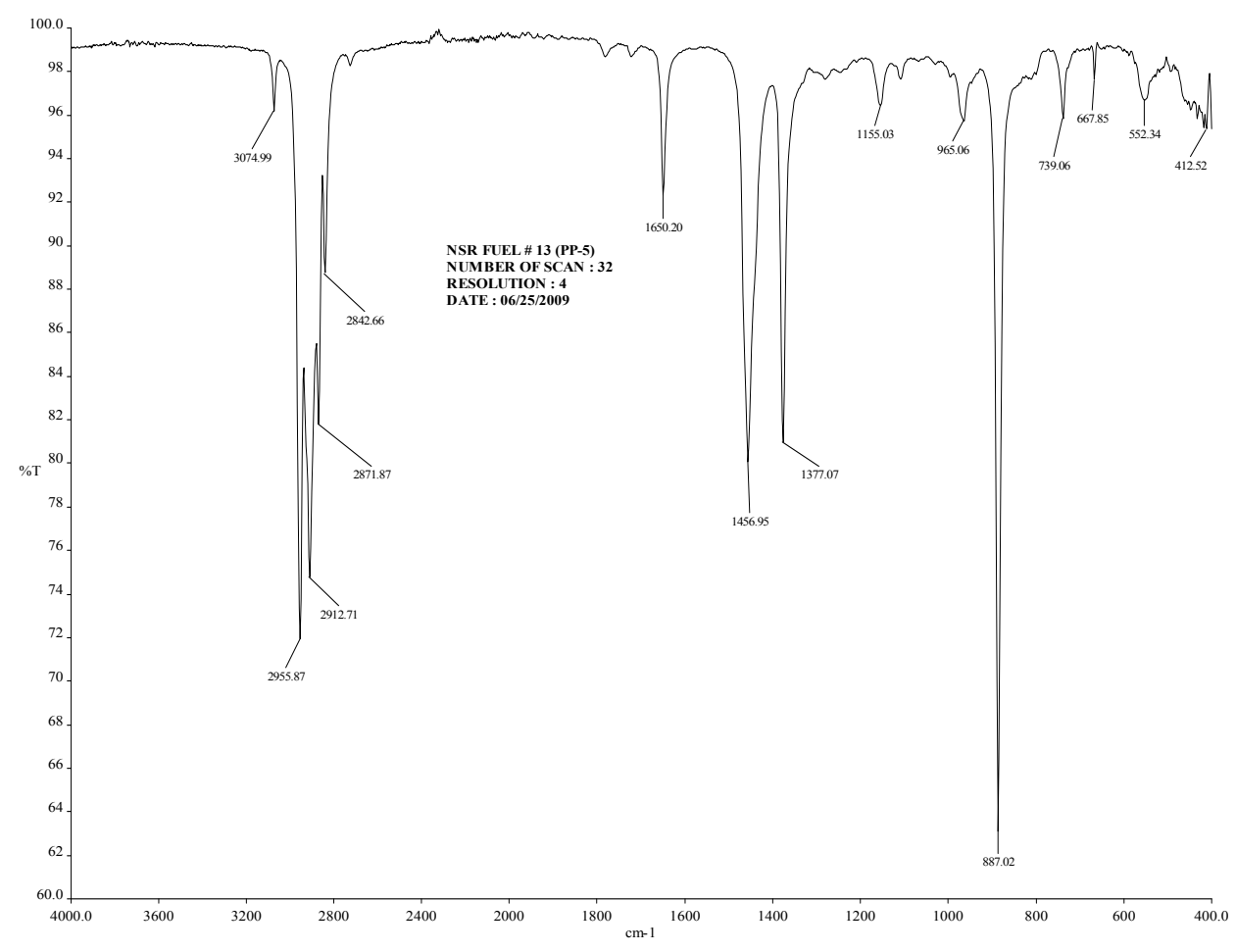

Fig. 18. FTIR Spectra of PP-5 Plastic to Fue.

\begin{tabular}{|c|c|c|c|c|c|}
\hline $\begin{array}{l}\text { Band Peak } \\
\text { Number }\end{array}$ & $\begin{array}{c}\text { Wave } \\
\text { Number } \\
\left(\mathrm{cm}^{-1}\right)\end{array}$ & $\begin{array}{l}\text { Compound } \\
\text { Group Name }\end{array}$ & $\begin{array}{l}\text { Band Peak } \\
\text { Number }\end{array}$ & $\begin{array}{c}\text { Wave } \\
\text { Number } \\
\left(\mathrm{cm}^{-1}\right)\end{array}$ & $\begin{array}{l}\text { Compound } \\
\text { Group Name }\end{array}$ \\
\hline 1 & 3074.99 & $\begin{array}{l}\text { H Bonded } \\
\text { NH }\end{array}$ & 8 & 1377.07 & $\mathrm{CH}_{3}$ \\
\hline 2 & 2955.87 & $\mathrm{C}-\mathrm{CH}_{3}$ & 9 & 1155.03 & \\
\hline 3 & 2912.71 & $\mathrm{C}-\mathrm{CH}_{3}$ & 10 & 965.06 & $\begin{array}{c}-\mathrm{CH}=\mathrm{CH}- \\
\text { (Trans) }\end{array}$ \\
\hline 4 & 2871.87 & $\mathrm{C}-\mathrm{CH}_{3}$ & 11 & 887.02 & $\mathrm{C}=\mathrm{CH}_{2}$ \\
\hline 5 & 2842.66 & $\mathrm{C}-\mathrm{CH}_{3}$ & 12 & 739.06 & $-\mathrm{CH}=\mathrm{CH}-(\mathrm{Cis})$ \\
\hline 6 & 1650.20 & Amides & 13 & 667.85 & $-\mathrm{CH}=\mathrm{CH}-(\mathrm{Cis})$ \\
\hline 7 & 1465.95 & $\mathrm{CH}_{2}$ & & & \\
\hline
\end{tabular}

Table 22. FTIR Spectra of PP-5 Plastic to Fuel Functional Group Name 


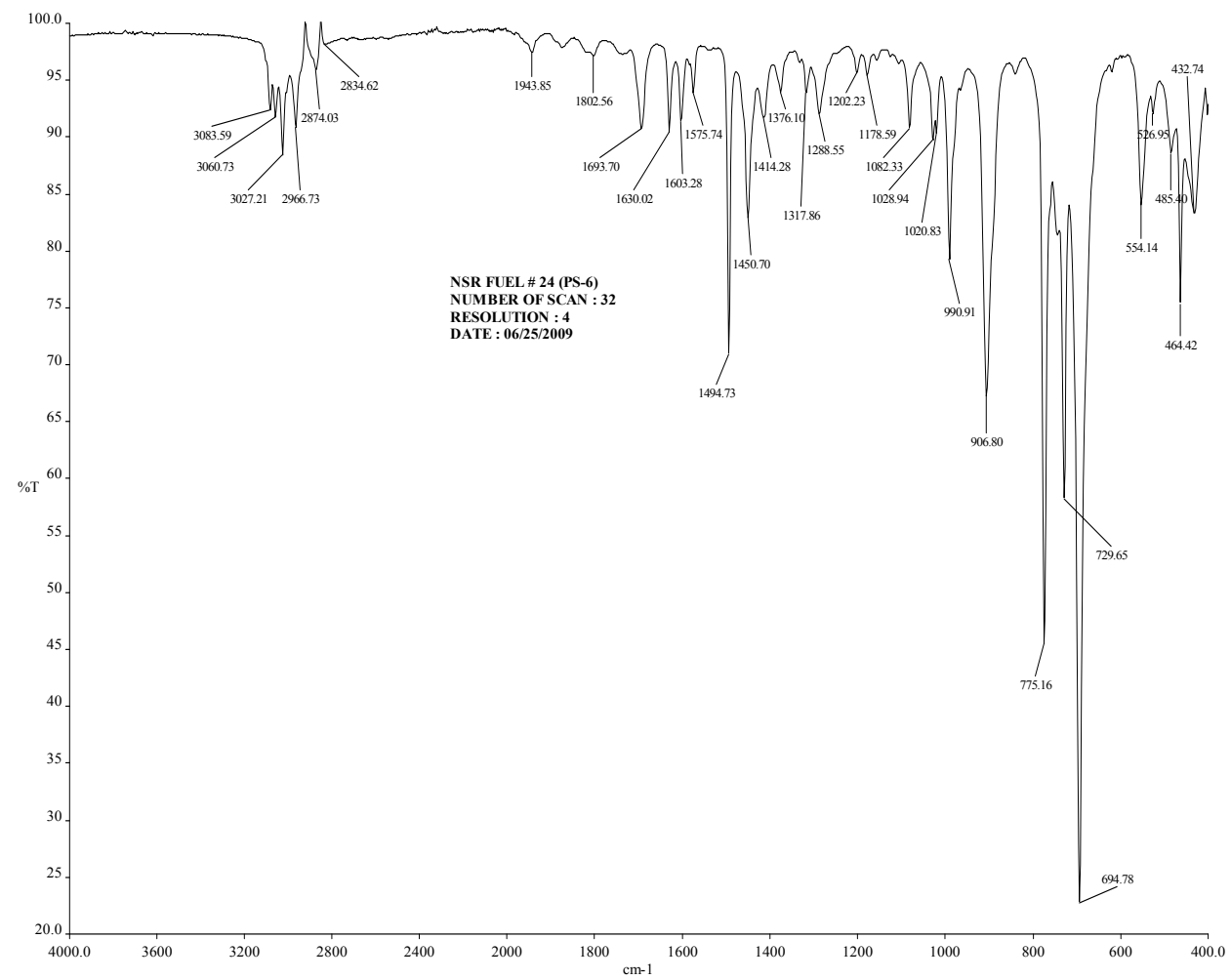

Fig. 19. FTIR Spectra of PS-6 Plastic to Fuel

\begin{tabular}{cccccc}
\hline $\begin{array}{c}\text { Band Peak } \\
\text { Number }\end{array}$ & $\begin{array}{c}\text { Wave } \\
\text { Number } \\
\left(\mathbf{c m}^{-1}\right)\end{array}$ & $\begin{array}{c}\text { Compound } \\
\text { Group Name }\end{array}$ & $\begin{array}{c}\text { Band Peak } \\
\text { Number }\end{array}$ & $\begin{array}{c}\text { Wave } \\
\text { Number } \\
\left(\mathbf{c m}^{-1} \mathbf{)}\right.\end{array}$ & $\begin{array}{c}\text { Compound } \\
\text { Group Name }\end{array}$ \\
\hline 1 & 3083.59 & $=\mathrm{C}-\mathrm{H}$ & 15 & 1414.28 & $\mathrm{CH}_{2}$ \\
2 & 3060.73 & $=\mathrm{C}-\mathrm{H}$ & 16 & 1376.10 & $\mathrm{CH}_{3}$ \\
3 & 3027.21 & $=\mathrm{C}-\mathrm{H}$ & 17 & 1317.86 & \\
4 & 2966.73 & $\mathrm{C}-\mathrm{CH}_{3}$ & 18 & 1288.55 & \\
5 & 2874.03 & $\mathrm{C}-\mathrm{CH}_{3}$ & 19 & 1202.23 & \\
6 & 2834.62 & $\mathrm{C}^{-\mathrm{CH}_{3}}$ & 20 & 1178.59 & \\
7 & 1943.85 & & 21 & 1082.33 & \\
8 & 1802.56 & Non-Conjugated & 22 & 1028.94 & Acetates \\
9 & 1693.70 & Conjugated & 23 & 1020.83 & Acetates \\
10 & 1630.02 & Conjugated & 24 & 990.91 & $-\mathrm{CH}=\mathrm{CH}_{2}$ \\
11 & 1603.28 & Conjugated & 25 & 906.80 & $-\mathrm{CH}=\mathrm{CH}$ \\
12 & 1575.74 & & 26 & 775.16 & \\
13 & 1494.73 & & 27 & 729.65 & $-\mathrm{CH}=\mathrm{CH}-(\mathrm{Cis})$ \\
14 & 1450.70 & $\mathrm{CH}_{3}$ & 28 & 694.78 & $-\mathrm{CH}=\mathrm{CH}-(\mathrm{Cis})$ \\
\hline
\end{tabular}

Table 23. FTIR Spectra of PS-6 Plastic to Fuel Functional Group Name 
In FTIR analysis of HDPE-2 fuel obtained functional groups are $\mathrm{C}_{-} \mathrm{CH}_{3}, \mathrm{CH}_{2}$, NonConjugated, $\mathrm{CH}_{3},-\mathrm{CH}=\mathrm{CH}_{2},-\mathrm{CH}=\mathrm{CH}-$ (Cis) and $-\mathrm{CH}=\mathrm{CH}-($ Trans) [Shown above, Fig.16\&Table-20].In LDPE-4 analysis functional groups are $\mathrm{C}_{-} \mathrm{CH}_{3}, \mathrm{CH}_{2}$, Non-Conjugated, $\mathrm{CH}_{3},-\mathrm{CH}=\mathrm{CH}_{2},-\mathrm{CH}=\mathrm{CH}-$ (Cis) and $-\mathrm{CH}=\mathrm{CH}-($ Trans) [Shown above, Fig.17 \&Table-21].In PP5 analysis functional groups are $\mathrm{CH}_{3}, \mathrm{C}-\mathrm{CH}_{2},-\mathrm{CH}=\mathrm{CH}-$ (Cis) and,- $\mathrm{CH}=\mathrm{CH}-$ (Trans). [Shown above, Fig.18 \&Table-22] Subsequently in PS-6 analysis obtained functional groups are $\mathrm{CH} 2$, $\mathrm{CH} 3$, Acetates, $-\mathrm{CH}=\mathrm{CH} 2$ and $-\mathrm{CH}=\mathrm{CH}$-(Cis) etc. [Shown above, Fig.19 \& Table-23].

\section{FTIR Analysis of Mixed Waste Plastics to Fuel:}

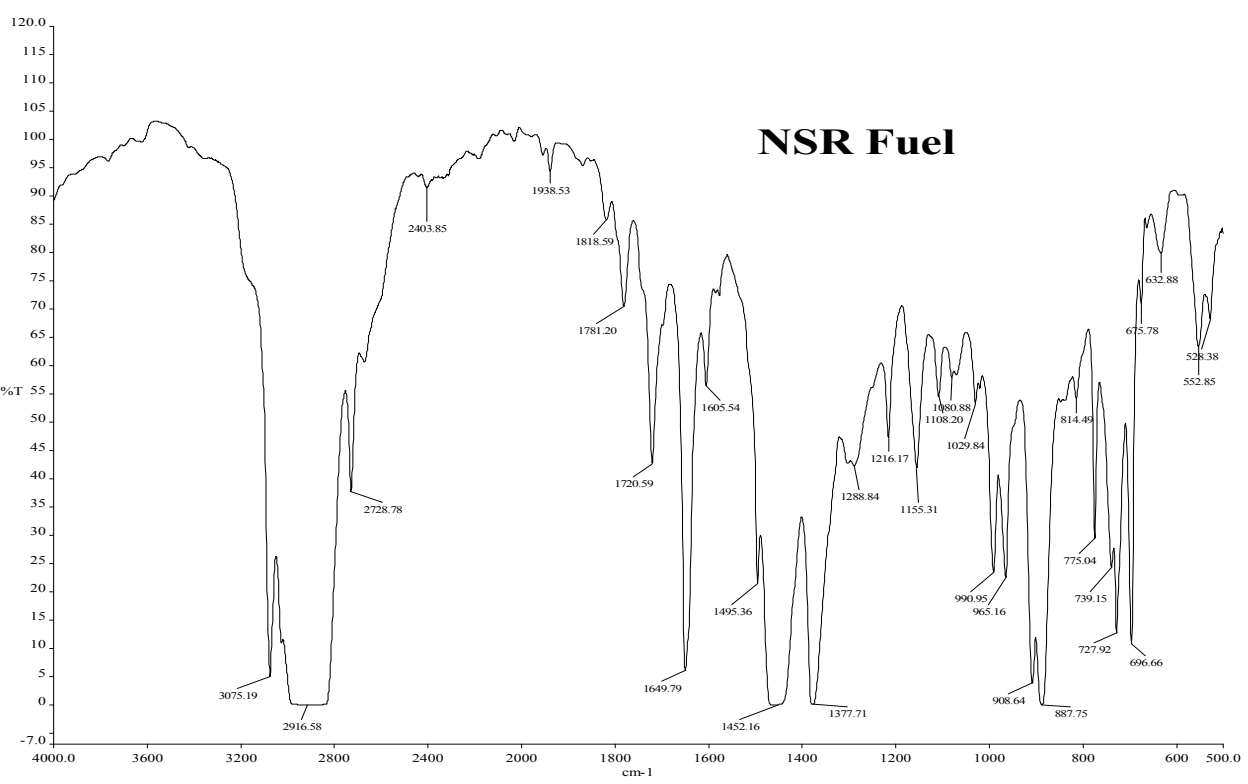

Fig. 20. FTIR Spectra of Mixed Waste Plastic to Fuel

\begin{tabular}{|c|c|c|c|c|c|}
\hline $\begin{array}{c}\text { Band Peak } \\
\text { Number }\end{array}$ & $\begin{array}{c}\text { Wave Number } \\
\left(\mathrm{cm}^{-1}\right)\end{array}$ & $\begin{array}{c}\text { Functional } \\
\text { Group Name }\end{array}$ & $\begin{array}{c}\text { Band Peak } \\
\text { Number }\end{array}$ & $\begin{array}{c}\text { Wave Number } \\
\left(\mathrm{cm}^{-1}\right)\end{array}$ & $\begin{array}{c}\text { Functional } \\
\text { Group Name }\end{array}$ \\
\hline 1 & 3075.19 & H Bonded NH & 13 & 1377.71 & $\mathrm{CH}_{3}$ \\
\hline 2 & 2916.58 & $\mathrm{CH}_{2}$ & 19 & 1029.84 & Acetates \\
\hline 3 & 2728.78 & $\mathrm{C}-\mathrm{CH}_{3}$ & 20 & 990.95 & $\begin{array}{c}\text { Secondary } \\
\text { Cyclic Alcohol }\end{array}$ \\
\hline 5 & 1938.53 & Non-Conjugated & 21 & 965.16 & $\begin{array}{c}-\mathrm{CH}=\mathrm{CH}- \\
\text { (trans) }\end{array}$ \\
\hline 6 & 1818.59 & Non-Conjugated & 22 & 908.64 & $-\mathrm{CH}=\mathrm{CH}_{2}$ \\
\hline 7 & 1781.20 & Non-Conjugated & 23 & 887.75 & $\mathrm{C}=\mathrm{CH}_{2}$ \\
\hline 8 & 1720.59 & Non-Conjugated & 26 & 739.15 & $-\mathrm{CH}=\mathrm{CH}-$ (cis) \\
\hline 9 & 1649.79 & Amides & 27 & 727.92 & $-\mathrm{CH}=\mathrm{CH}-(\mathrm{cis})$ \\
\hline 10 & 1605.54 & Non-Conjugated & 28 & 696.66 & $-\mathrm{CH}=\mathrm{CH}-$ (cis) \\
\hline 12 & 1452.16 & $\mathrm{CH}_{2}$ & 29 & 675.78 & $-\mathrm{CH}=\mathrm{CH}-(\mathrm{cis})$ \\
\hline
\end{tabular}

Table 24. FTIR Spectra of Mixed Waste Plastic to Fuel Functional Group Name 
In FTIR analysis of mixed waste plastics to NSR fuel obtained functional groups are: $\mathrm{CH}_{3}$, Acetates, Secondary Cyclic Alcohol, $-\mathrm{CH}=\mathrm{CH}_{2}, \mathrm{C}=\mathrm{CH}_{2},-\mathrm{CH}=\mathrm{CH}-(\mathrm{Cis})$ and $-\mathrm{CH}=\mathrm{CH}-($ Trans $)$ etc. [Shown above, Fig. 20 \&Table-24].

FTIR Analysis of Mixed Waste Plastics to Fractional Distillation Fuel:

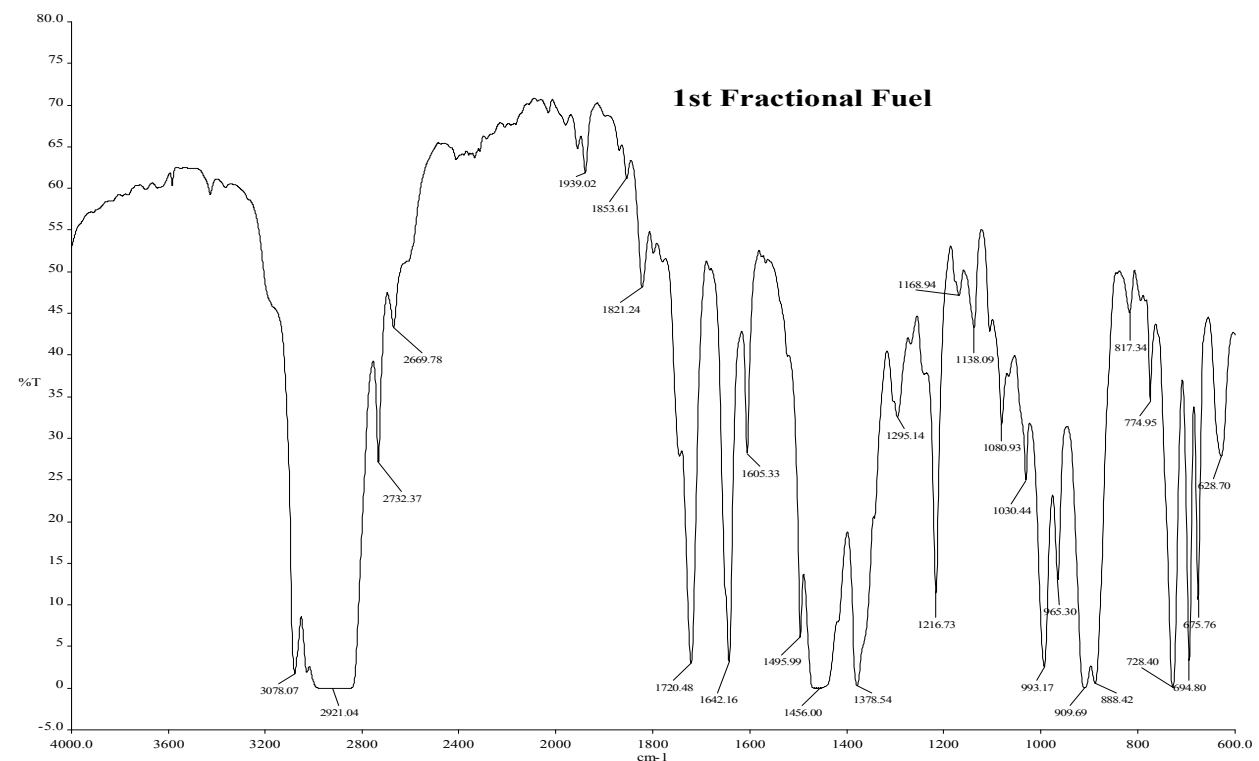

Fig. 21. FTIR Spectra of Mixed Waste Plastic Fuel to $1^{\text {st }}$ Fractional Fuel (Gasoline)

\begin{tabular}{|c|c|c|c|c|c|}
\hline $\begin{array}{l}\text { Band Peak } \\
\text { Number }\end{array}$ & $\begin{array}{c}\text { Wave } \\
\text { Number } \\
\left(\mathrm{cm}^{-1}\right)\end{array}$ & $\begin{array}{l}\text { Functional } \\
\text { Group Name }\end{array}$ & $\begin{array}{c}\text { Band Peak } \\
\text { Number }\end{array}$ & $\begin{array}{c}\text { Wave } \\
\text { Number } \\
\left(\mathrm{cm}^{-1}\right)\end{array}$ & $\begin{array}{l}\text { Functional } \\
\text { Group Name }\end{array}$ \\
\hline 1 & 3078.07 & H Bonded NH & 13 & 1378.54 & $\mathrm{CH}_{3}$ \\
\hline 2 & 2921.04 & $\mathrm{C}-\mathrm{CH}_{3}$ & 19 & 1030.44 & Acetates \\
\hline 3 & 2732.37 & $\mathrm{C}-\mathrm{CH}_{3}$ & 20 & 993.17 & $\begin{array}{c}\text { Secondary } \\
\text { Cyclic Alcohol }\end{array}$ \\
\hline 4 & 2669.78 & $\mathrm{C}-\mathrm{CH}_{3}$ & 21 & 965.30 & $\begin{array}{c}-\mathrm{CH}=\mathrm{CH}- \\
\text { (trans) }\end{array}$ \\
\hline 6 & 1853.61 & $\begin{array}{c}\text { Non- } \\
\text { Conjugated }\end{array}$ & 22 & 909.69 & $-\mathrm{CH}=\mathrm{CH}_{2}$ \\
\hline 7 & 1821.24 & $\begin{array}{c}\text { Non- } \\
\text { Conjugated }\end{array}$ & 23 & 888.42 & $\mathrm{C}=\mathrm{CH}_{2}$ \\
\hline 8 & 1720.48 & $\begin{array}{c}\text { Non- } \\
\text { Conjugated }\end{array}$ & 26 & 728.40 & $-\mathrm{CH}=\mathrm{CH}-(\mathrm{cis})$ \\
\hline 9 & 1642.16 & Conjugated & 27 & 694.80 & $-\mathrm{CH}=\mathrm{CH}-(\mathrm{cis})$ \\
\hline 10 & 1605.33 & Conjugated & 28 & 675.76 & $-\mathrm{CH}=\mathrm{CH}-$ (cis) \\
\hline 12 & 1456.00 & $\mathrm{CH}_{3}$ & 29 & 628.70 & $-\mathrm{CH}=\mathrm{CH}-(\mathrm{cis})$ \\
\hline
\end{tabular}

Table 25. Mixed Waste Plastic Fuel to $1^{\text {st }}$ Fractional Fuel (Gasoline) FTIR Functional Group List 


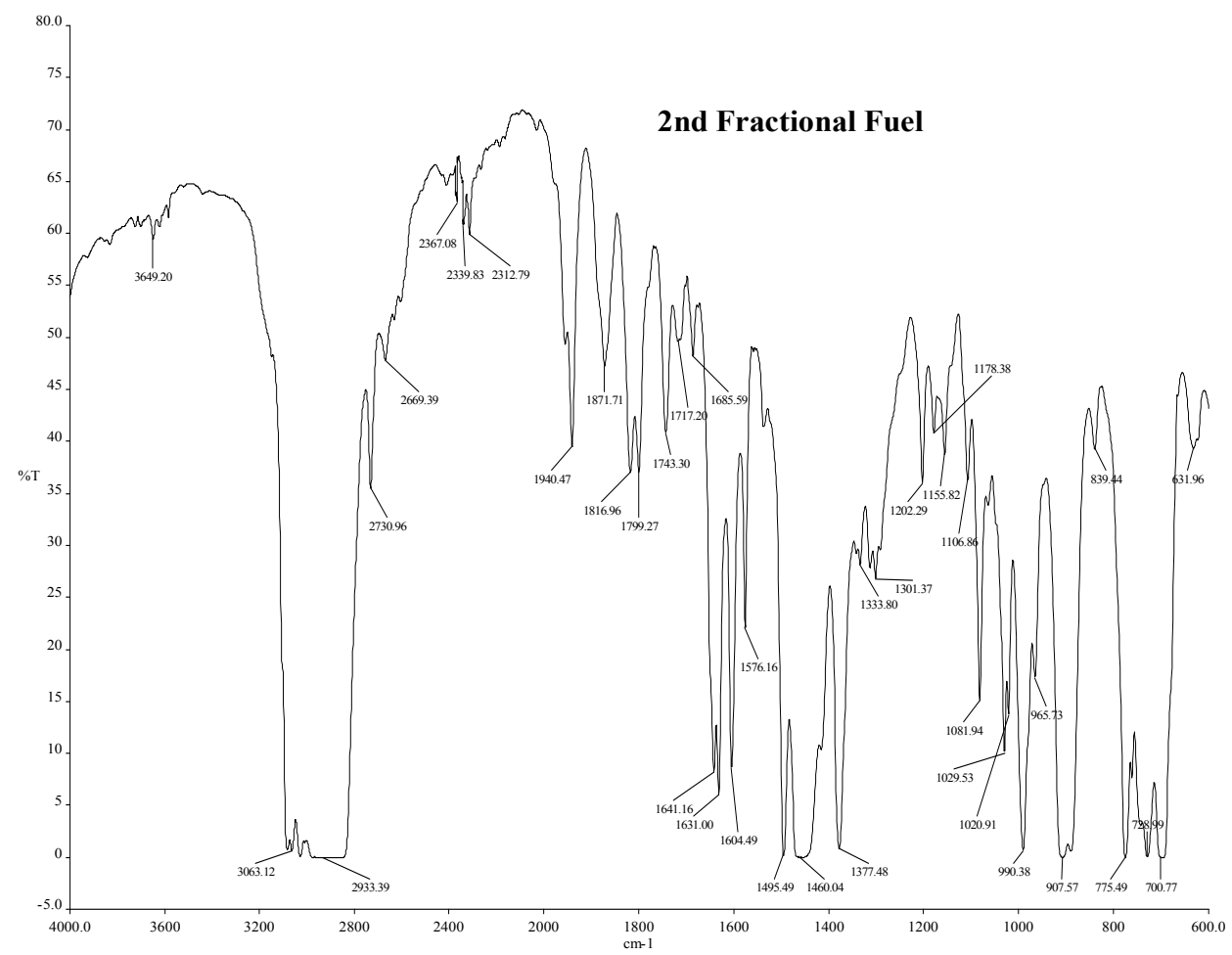

Fig. 22. FTIR Spectra of Mixed Waste Plastic Fuel to $2^{\text {nd }}$ Fractional Fuel (Naphtha, Chemical)

\begin{tabular}{|c|c|c|c|c|c|}
\hline $\begin{array}{c}\text { Band Peak } \\
\text { Number }\end{array}$ & $\begin{array}{c}\text { Wave } \\
\text { Number } \\
\left(\mathrm{cm}^{-1}\right)\end{array}$ & $\begin{array}{l}\text { Functional } \\
\text { Group Name }\end{array}$ & $\begin{array}{c}\text { Band Peak } \\
\text { Number }\end{array}$ & $\begin{array}{c}\text { Wave } \\
\text { Number } \\
\left(\mathrm{cm}^{-1}\right)\end{array}$ & $\begin{array}{l}\text { Functional } \\
\text { Group Name }\end{array}$ \\
\hline 2 & 3063.12 & $=\mathrm{C}-\mathrm{H}$ & 16 & 1641.16 & $\begin{array}{c}\text { Non- } \\
\text { Conjugated }\end{array}$ \\
\hline 3 & 2933.39 & $\mathrm{C}-\mathrm{CH}_{3}$ & 17 & 1631.00 & $\begin{array}{c}\text { Non- } \\
\text { Conjugated }\end{array}$ \\
\hline 4 & 2730.96 & $\mathrm{C}-\mathrm{CH}_{3}$ & 21 & 1460.04 & $\mathrm{CH}_{3}$ \\
\hline 5 & 2669.39 & $\mathrm{C}-\mathrm{CH}_{3}$ & 22 & 1377.48 & $\mathrm{CH}_{3}$ \\
\hline 9 & 1940.47 & Non-Conjugated & 30 & 1029.53 & Acetates \\
\hline 10 & 1871.71 & Non-Conjugated & 31 & 1020.91 & Acetates \\
\hline 11 & 1816.96 & Non-Conjugated & 32 & 990.38 & $-\mathrm{CH}=\mathrm{CH}_{2}$ \\
\hline 12 & 1799.27 & Non-Conjugated & 33 & 965.73 & $\begin{array}{c}-\mathrm{CH}=\mathrm{CH}- \\
\text { (trans) }\end{array}$ \\
\hline 13 & 1743.30 & Conjugated & 34 & 907.57 & $-\mathrm{CH}=\mathrm{CH}_{2}$ \\
\hline 14 & 1717.20 & Non-Conjugated & 37 & 728.99 & $-\mathrm{CH}=\mathrm{CH}-(\mathrm{cis})$ \\
\hline 15 & 1685.59 & Conjugated & 38 & 700.77 & $-\mathrm{CH}=\mathrm{CH}-(\mathrm{cis})$ \\
\hline
\end{tabular}

Table 26. Mixed Waste Plastic Fuel to 2nd Fractional Fuel (Naphtha) FTIR Functional Group List 


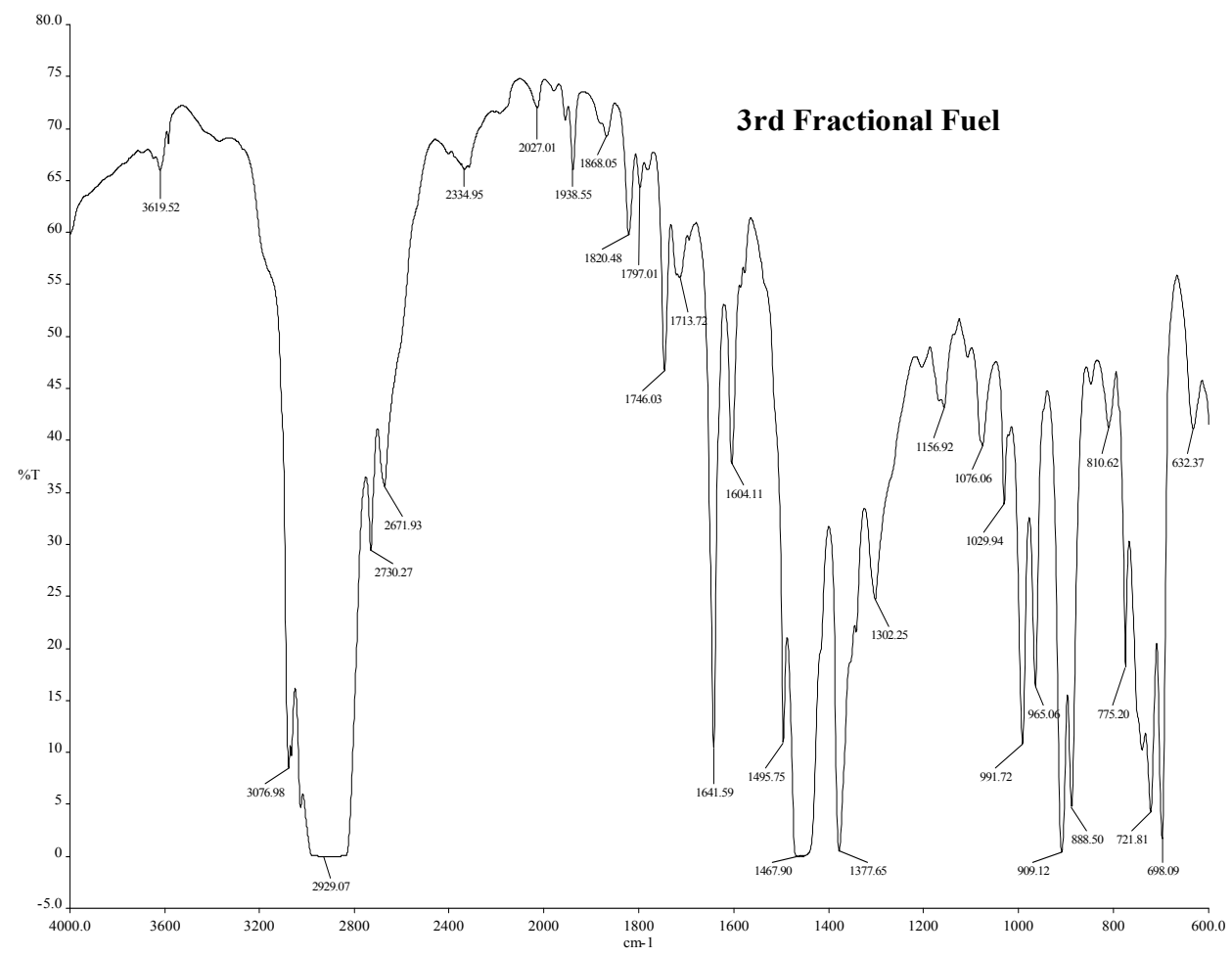

Fig. 23. FTIR Spectra of Mixed Waste Plastic Fuel to 3rd Fractional Fuel (Aviation)

\begin{tabular}{|c|c|c|c|c|c|}
\hline $\begin{array}{c}\text { Band Peak } \\
\text { Number }\end{array}$ & $\begin{array}{c}\text { Wave } \\
\text { Number } \\
\left(\mathrm{cm}^{-1}\right)\end{array}$ & $\begin{array}{l}\text { Functional } \\
\text { Group Name }\end{array}$ & $\begin{array}{c}\text { Band Peak } \\
\text { Number }\end{array}$ & $\begin{array}{c}\text { Wave } \\
\text { Number } \\
\left(\mathrm{cm}^{-1}\right)\end{array}$ & $\begin{array}{l}\text { Functional } \\
\text { Group Name }\end{array}$ \\
\hline 3 & 2929.07 & $\mathrm{C}-\mathrm{CH}_{3}$ & 17 & 1467.90 & $\mathrm{CH}_{3}$ \\
\hline 4 & 2730.27 & $\mathrm{C}-\mathrm{CH}_{3}$ & 18 & 1377.65 & $\mathrm{CH}_{3}$ \\
\hline 5 & 2671.93 & $\mathrm{C}-\mathrm{CH}_{3}$ & 22 & 1029.94 & Acetates \\
\hline 8 & 1938.55 & Non-Conjugated & 23 & 991.72 & $-\mathrm{CH}=\mathrm{CH}_{2}$ \\
\hline 9 & 1868.05 & Non-Conjugated & 24 & 965.06 & $\begin{array}{c}-\mathrm{CH}=\mathrm{CH}- \\
\text { (trans) }\end{array}$ \\
\hline 10 & 1820.48 & Non-Conjugated & 25 & 909.12 & $\mathrm{CH}=\mathrm{CH}_{2}$ \\
\hline 11 & 1797.01 & Non-Conjugated & 26 & 888.50 & $\mathrm{C}=\mathrm{CH}_{2}$ \\
\hline 12 & 1746.03 & Non-Conjugated & 29 & 721.81 & $-\mathrm{CH}=\mathrm{CH}-(\mathrm{cis})$ \\
\hline 13 & 1713.72 & Non-Conjugated & 30 & 698.09 & $-\mathrm{CH}=\mathrm{CH}-(\mathrm{cis})$ \\
\hline 14 & 1641.59 & Non-Conjugated & & & \\
\hline
\end{tabular}

Table 27. Mixed Waste Plastic Fuel to $3 \mathrm{rd}^{\mathrm{t}}$ Fractional Fuel (Aviation) FTIR Functional Group List 


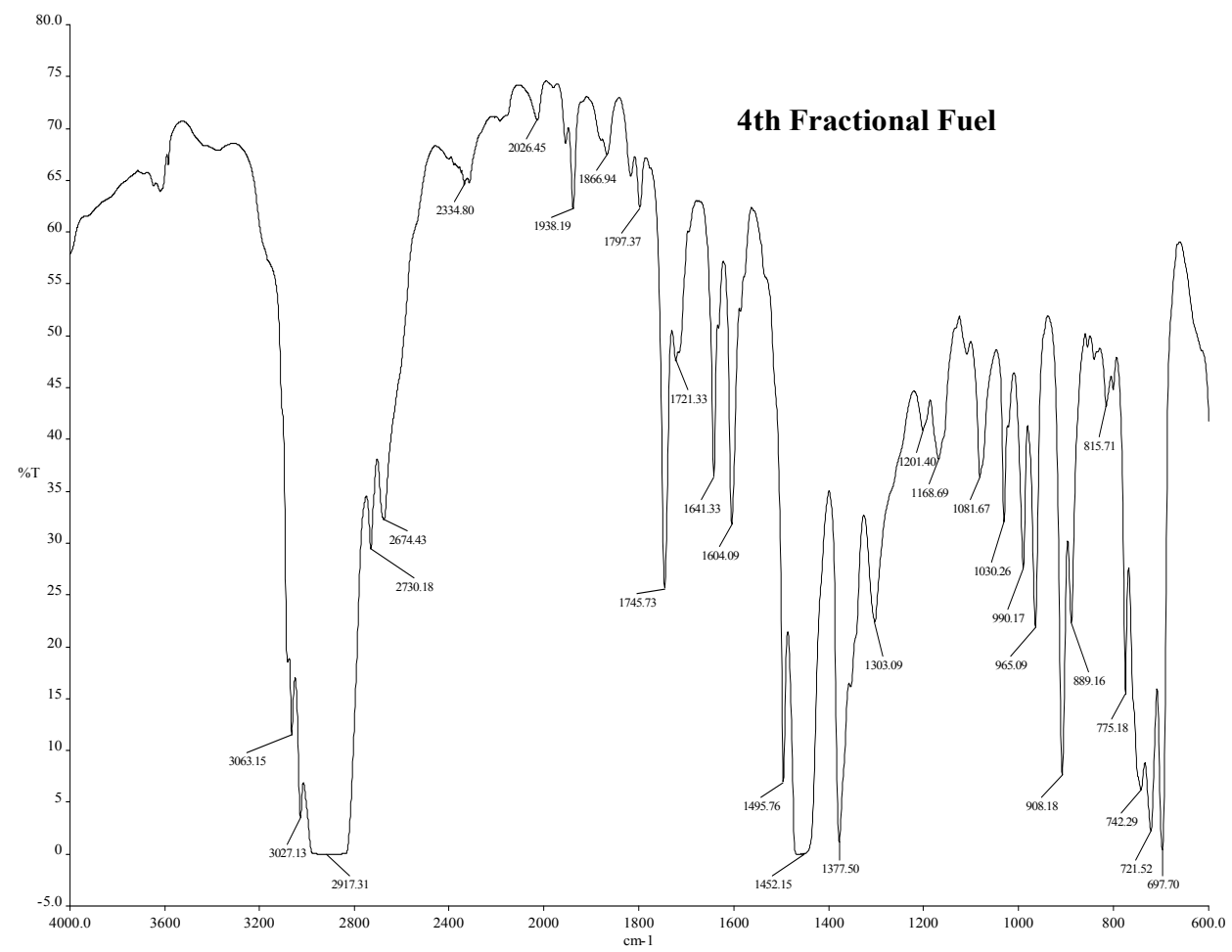

Fig. 24. FTIR Spectra of Mixed Waste Plastic to Fuel (Diesel)

\begin{tabular}{|c|c|c|c|c|c|}
\hline $\begin{array}{c}\text { Band Peak } \\
\text { Number }\end{array}$ & $\begin{array}{c}\text { Wave } \\
\text { Number } \\
\left(\mathrm{cm}^{-1}\right)\end{array}$ & $\begin{array}{l}\text { Functional } \\
\text { Group Name }\end{array}$ & $\begin{array}{c}\text { Band Peak } \\
\text { Number }\end{array}$ & $\begin{array}{c}\text { Wave } \\
\text { Number } \\
\left(\mathrm{cm}^{-1}\right)\end{array}$ & $\begin{array}{l}\text { Functional } \\
\text { Group Name }\end{array}$ \\
\hline 1 & 3063.15 & $=\mathrm{C}-\mathrm{H}$ & 16 & 1452.15 & $\mathrm{CH}_{2}$ \\
\hline 2 & 3027.13 & $=\mathrm{C}-\mathrm{H}$ & 17 & 1377.50 & $\mathrm{CH}_{3}$ \\
\hline 3 & 2917.31 & $\mathrm{CH}_{2}$ & 22 & 1030.26 & Acetates \\
\hline 4 & 2730.18 & $\mathrm{C}-\mathrm{CH}_{3}$ & 23 & 990.17 & $-\mathrm{CH}=\mathrm{CH}_{2}$ \\
\hline 5 & 2674.43 & $\mathrm{C}-\mathrm{CH}_{3}$ & 24 & 965.09 & $\begin{array}{c}-\mathrm{CH}=\mathrm{CH}- \\
\text { (trans) }\end{array}$ \\
\hline 8 & 1938.19 & Non-Conjugated & 25 & 908.18 & $-\mathrm{CH}=\mathrm{CH}_{2}$ \\
\hline 9 & 1866.94 & Non-Conjugated & 26 & 889.16 & $\mathrm{C}=\mathrm{CH}_{2}$ \\
\hline 10 & 1797.37 & Non-Conjugated & 29 & 742.29 & $-\mathrm{CH}=\mathrm{CH}-(\mathrm{cis})$ \\
\hline 11 & 1745.73 & Non-Conjugated & 30 & 721.52 & $-\mathrm{CH}=\mathrm{CH}-$ (cis) \\
\hline 12 & 1721.33 & Non-Conjugated & 31 & 697.70 & $-\mathrm{CH}=\mathrm{CH}-(\mathrm{cis})$ \\
\hline 13 & 1641.33 & Non-Conjugated & & & \\
\hline
\end{tabular}

Table 28. Mixed Waste Plastic Fuel to 4th Fractional Fuel (Diesel) FTIR Functional Group List 


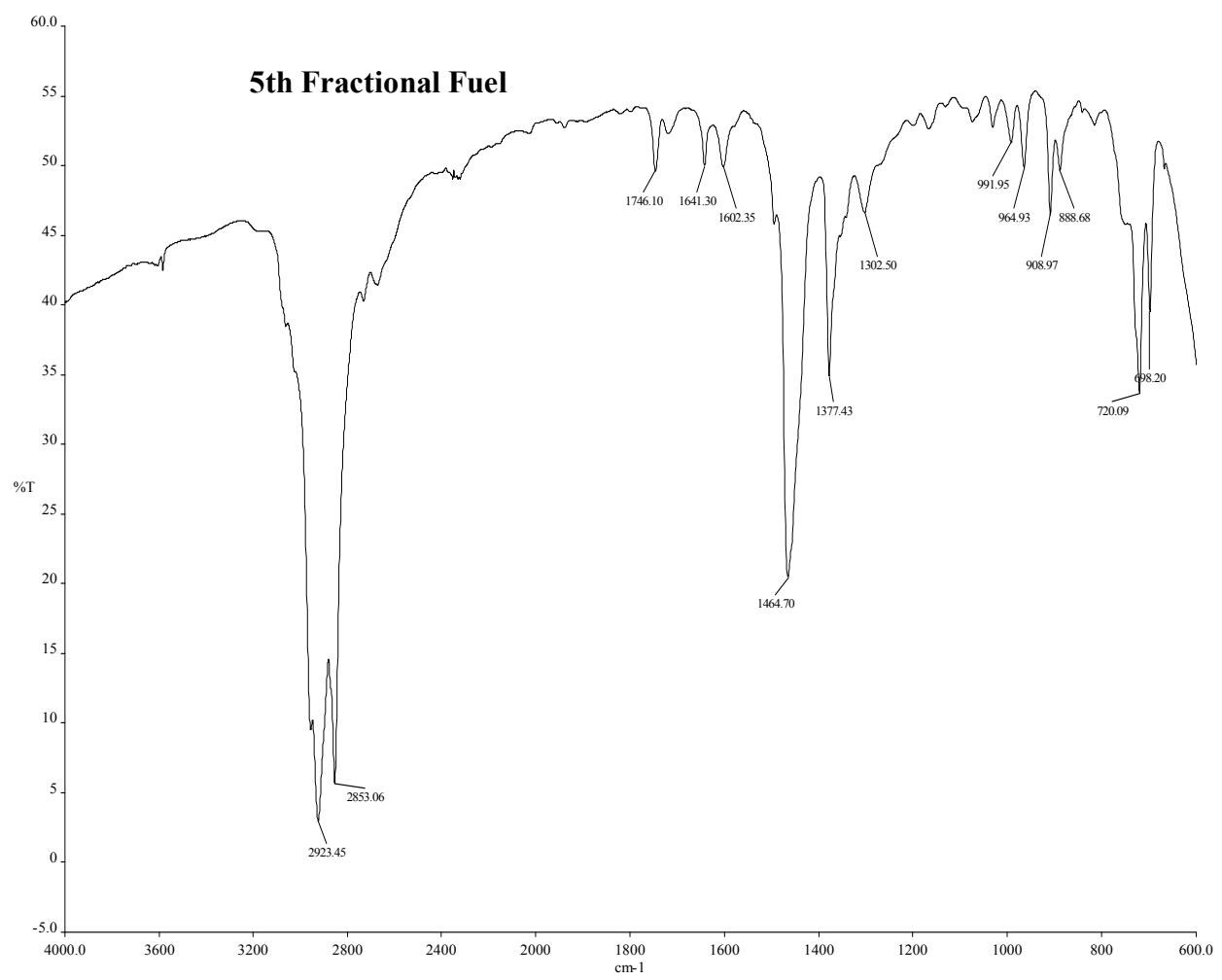

Fig. 25. FTIR Spectra of Mixed Waste Plastic to Fuel (Fuel Oil)

\begin{tabular}{|c|c|c|c|c|c|}
\hline $\begin{array}{c}\text { Band Peak } \\
\text { Number }\end{array}$ & $\begin{array}{c}\text { Wave } \\
\text { Number } \\
\left(\mathrm{cm}^{-1}\right)\end{array}$ & $\begin{array}{l}\text { Functional } \\
\text { Group Name }\end{array}$ & $\begin{array}{c}\text { Band Peak } \\
\text { Number }\end{array}$ & $\begin{array}{c}\text { Wave } \\
\text { Number } \\
\left(\mathrm{cm}^{-1}\right)\end{array}$ & $\begin{array}{l}\text { Functional } \\
\text { Group Name }\end{array}$ \\
\hline 1 & 2923.45 & $\mathrm{CH}_{2}$ & 9 & 991.95 & $\begin{array}{c}\text { Secondary } \\
\text { Cyclic Alcohol }\end{array}$ \\
\hline 2 & 2853.06 & $\mathrm{CH}_{2}$ & 10 & 964.93 & $\begin{array}{l}-\mathrm{CH}=\mathrm{CH}- \\
\quad(\text { trans) }\end{array}$ \\
\hline 3 & 1746.10 & Non-Conjugated & 11 & 908.97 & $-\mathrm{CH}=\mathrm{CH}_{2}$ \\
\hline 4 & 1641.30 & Non-Conjugated & 12 & 888.68 & $\mathrm{C}=\mathrm{CH}_{2}$ \\
\hline 5 & 1602.35 & Non-Conjugated & 13 & 720.09 & $-\mathrm{CH}=\mathrm{CH}-(\mathrm{cis})$ \\
\hline 6 & 1464.70 & $\mathrm{CH}_{2}$ & 14 & 698.20 & $-\mathrm{CH}=\mathrm{CH}-(\mathrm{cis})$ \\
\hline 7 & 1377.43 & $\mathrm{CH}_{3}$ & & & \\
\hline
\end{tabular}

Table 29. Mixed Waste Plastic Fuel to 5th Fractional Fuel (Fuel Oil) FTIR Functional Group List

In FTIR analysis of fractional distillation fuel such as in 1ST Fraction Fuel (Gasoline) obtained functional groups are $\mathrm{CH}_{3}$, Acetates, Secondary Cyclic Alcohol, $-\mathrm{CH}=\mathrm{CH}_{2}$, 
$\mathrm{C}=\mathrm{CH}_{2}$,nad $-\mathrm{CH}=\mathrm{CH}-$ (Cis). [Shown above, Fig.21 \& Table-25]. In 2nd Fraction Fuel (Naphtha) analysis functional groups are $\mathrm{CH}_{3}$, Non-Conjugated, Acetates, $\mathrm{CH}=\mathrm{CH}_{2}$,$\mathrm{CH}=\mathrm{CH}-(\mathrm{Cis})$ and $-\mathrm{CH}=\mathrm{CH}-\left(\right.$ Trans). [Shown above, Fig.22\&Table-26]. In $3^{\text {rd }}$ Fraction Fuel (Aviation) analysis functional groups are $\mathrm{CH}_{3}$, Acetates, $\mathrm{C}-\mathrm{CH}_{2}, \mathrm{CH}=\mathrm{CH}-$ (Cis) and $\mathrm{CH}=\mathrm{CH}$-(Trans) [Shown above, Fig.23\&Table-27]. In $4^{\text {th }}$ Fraction Fuel (Diesel) analysis functional groups are $\mathrm{CH}_{2}, \mathrm{CH}_{3}$, Acetates, $-\mathrm{CH}=\mathrm{CH}_{2}, \mathrm{C}=\mathrm{CH}_{2}$ and, $-\mathrm{CH}=\mathrm{CH}$ - (Cis) [Shown above, Fig.24 \&Table-28]. Subsequently in $5^{\text {th }}$ Fraction Fuel (Fuel Oil) analysis obtained functional groups are Secondary Cyclic Alcohol, $-\mathrm{CH}=\mathrm{CH}_{2}, \mathrm{C}=\mathrm{CH}_{2},-\mathrm{CH}=\mathrm{CH}$ (Trans) and $\mathrm{CH}=\mathrm{CH}-(\mathrm{Cis})$ etc. [Shown above, Fig.25\&Table-29].

\section{Electricity production from waste plastic fuel}

Both NSR fractional fuels (NSR fractional 1st Fractional Fuel and NSR 4th Fractional Fuel) have been used to produce electricity by the help of conventional internal combustion generator. A flow diagram illustrating the process of energy production and consumption from NSR Fuel (Heating Oil) is shown below in Fig.26.

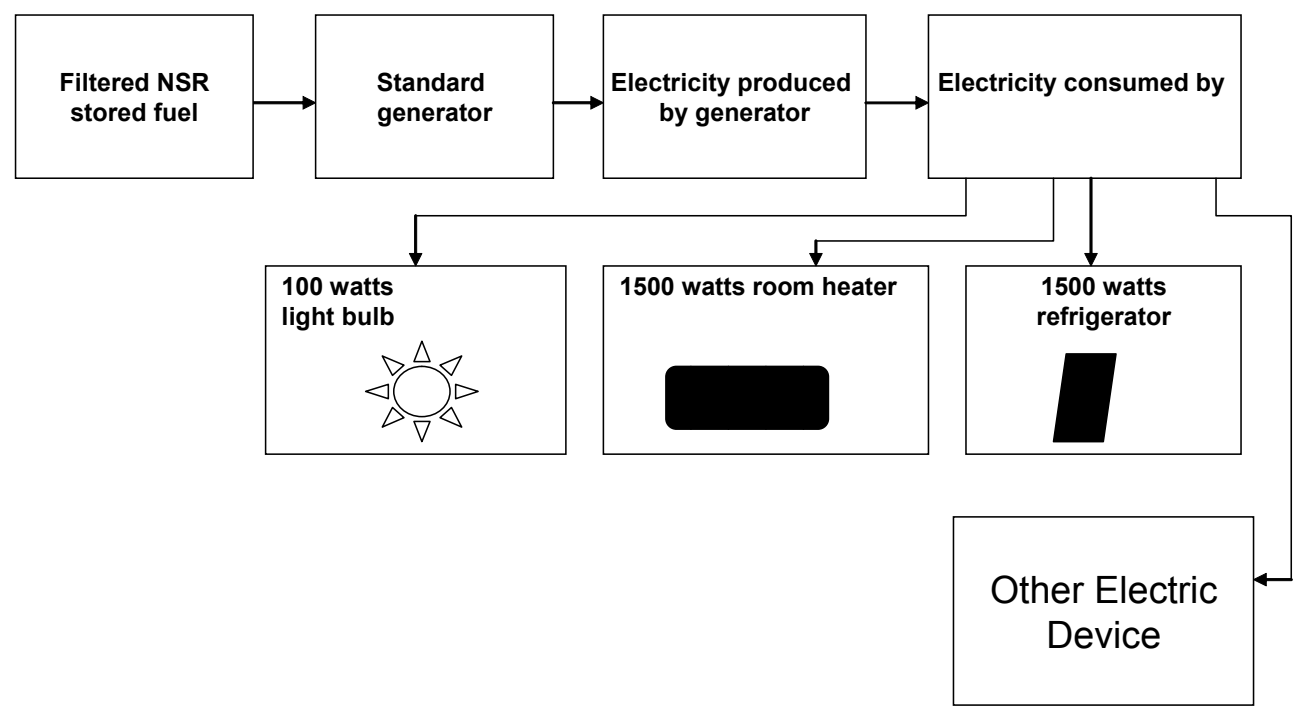

Fig. 26. Flow diagram of electricity generation consumption

NSR fractional 1st collection fuel was used in a gasoline generator with max $4.0 \mathrm{~kW}$ and volt output of 120. $\sim 1$ litter of fractional fuel was injected in the generator and with $\sim 2900$ watt constant demand; the generator ran a total of 42 minutes. A similar test was performed with commercial gasoline (87). $\sim 1$ litter of commercial gasoline (87) was injected and with the same $\sim 2900$ watt, constant demand the generator ran a total of 38 minutes. The difference in time occurs because NSR fraction 1st collection fuel has longer Carbon content than that of the commercial gasoline (87).

NSR fractional 4th collection fuel was used in a diesel generator with a max $4.0 \mathrm{~kW}$ and an output of 120 volt. $\sim 1$ litter of NSR fractional $2^{\text {nd }}$ collection fuel was injected in the generator and with a constant demand of 3200 watt; the generator ran a total of 42 minutes. The same 
test was conducted with commercial diesel, and with the same demand the generator ran for 34 minutes.

A diagram [Fig.27] is provided below showing the produced electricity consumption of commercial gasoline (87) and NSR fractional fuel 1st collection.

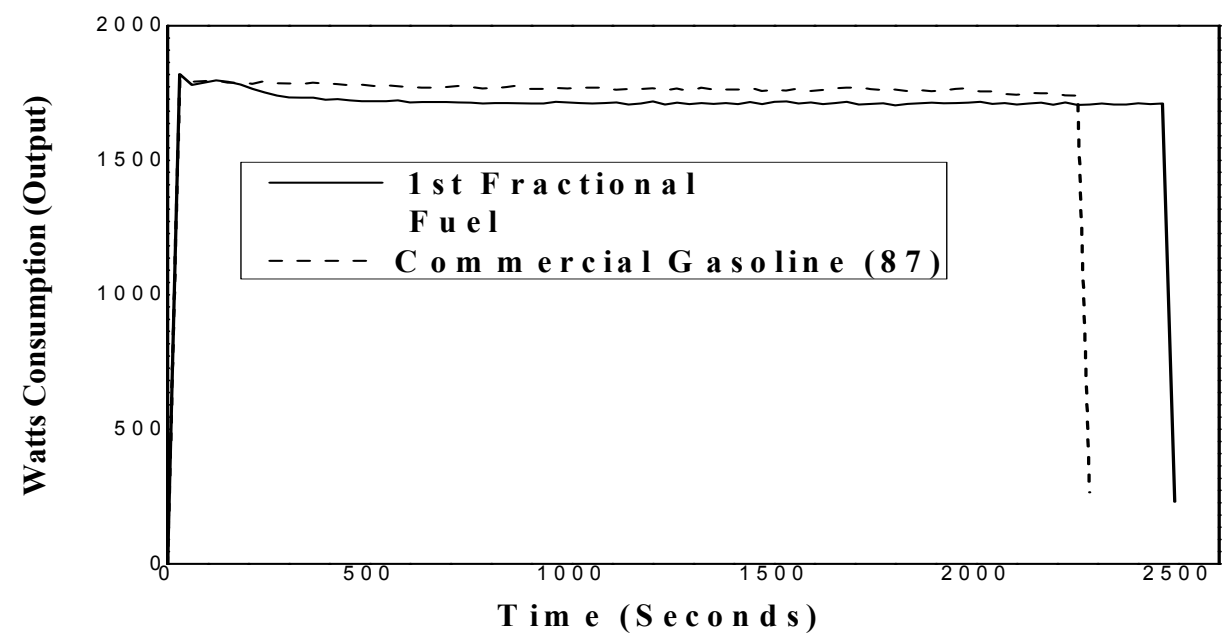

Fig. 27. Electricity Consumption and run time monitored by EML 2020 logger system for $1^{\text {st }}$ Fractional Fuel (Gasoline) and Commercial Gasoline87.

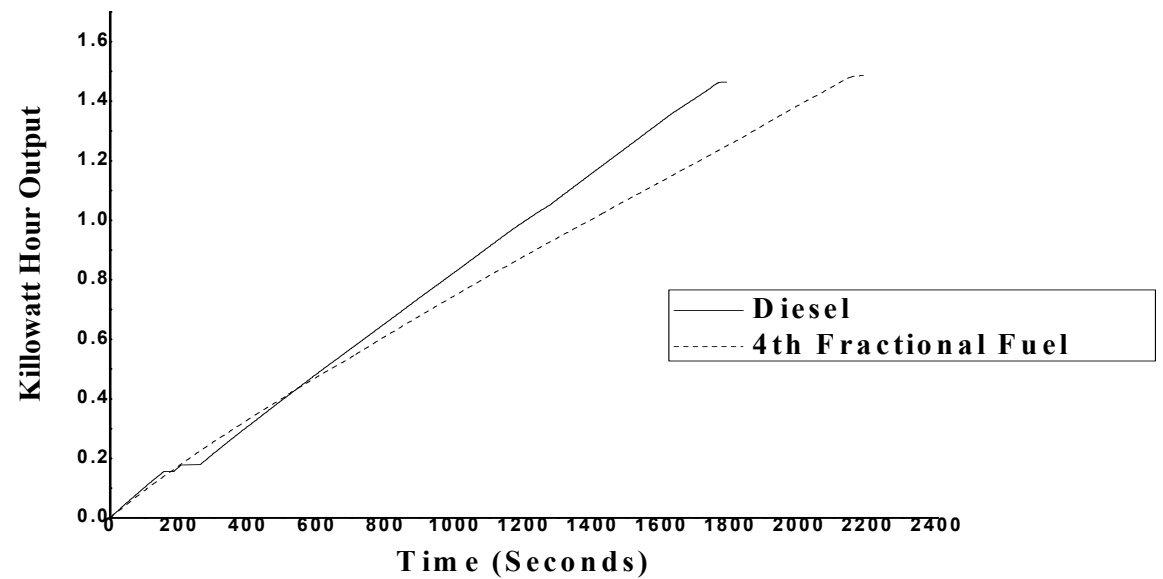

Fig. 28. Electricity Output Comparison Graph of Waste Plastic Fuel to $4^{\text {th }}$ Fractional Fuel and Commercial Diesel Fuel 


\begin{tabular}{ccccc}
\hline $\begin{array}{c}\text { Fuel Name } \\
4^{\text {th }} \text { Fractional Fuel (Diesel) }\end{array}$ & Generator & Fuel Amount & Duration & $\mathbf{k W h}$ \\
& AMCO & 1 Liter & $37 \mathrm{~min}$ & 2.028 \\
\hline Commercial Diesel & AMCO & 1 Liter & $28 \mathrm{~min}$ & 1.463 \\
\hline
\end{tabular}

Table 30. Comparison Table of $4^{\text {th }}$ Fractional Fuel (Diesel) and Commercial Diesel

Comparison of NSR $4^{\text {th }}$ fraction fuel and commercial diesel was conduced using an AMCO Diesel Generator. Above, Fig. 28 and Table 30 demonstrate the comparative results between the two fuels. The results indicate that the NSR-2 fuel provided a longer run time of the generator than the diesel. This is due to the NSR fuel having longer carbon chains than the diesel fuel.

\section{Automobile test driving}

Both NSR fractional $5^{\text {th }}$ collection fuel and commercial gasoline (87) was used for a comparison automobile test. A 1984 Oldsmobile vehicle (V-8 powered engine) was used for the test-drive and one gallon of fuel was used for both cases after complete drainage of the pre-existing fuel in the fuel tank. The test-drive was done on a rural highway with an average speed of $55 \mathrm{mph}$.

Based on the preliminary automobile test-drive, the NSR fuel has offered a competitive advantage in mileage over the commercial gasoline-87. NSR fuel showed better mileage performance of 21 miles per gallon (mpg) compared to $18 \mathrm{mpg}$ with commercial gasoline (87).

It is expected that NSR double condensed fuel will show even higher performance with more fuel-efficient car such as V-4 engine and hybrid vehicles. Additional test-driving is going to be conducted in the near future to verify the results.

\section{Conclusion}

The conversion of municipal waste plastics to liquid hydrocarbon fuel was carried out in thermal degradation process with/without catalyst. Individually we ran our experiment on waste plastics such as: HDPE-2, LDPE-4, PP-5 \& PS-6. Each of those experiment procedures are maintained identically, every ten (10) minutes of interval experiment was monitored and found during the condensation time changes of individual waste plastics external behavior different because of their different physical and chemical properties. Similarly, we ran another experiment with $2 \mathrm{~kg}$ of mixture of waste plastics in stainless steel reactor. Initial temperature is $350{ }^{\circ} \mathrm{C}$ for quick melting and optimum temperature is $305{ }^{\circ} \mathrm{C}$. For glass reactor every experiment temperature was maintained by variac meter, when experiment started variac percent was $90 \%$ (Tem- $405{ }^{\circ} \mathrm{C}$ ) for quick melting, after melted variac percent decreased to $70 \%$ (Tem- $315{ }^{\circ} \mathrm{C}$ ) due to smoke formation. Average (optimum) used variac percent in this experiment $75 \%\left(337.5^{\circ} \mathrm{C}\right)$. Gradually temperature range was maintained by variacmeter with proper monitoring. In fractional distillation process we separated different category of fuel such as gasoline, naphtha, jet fuel, diesel and fuel oil in accordance with their boiling point temperature profile. 


\section{Acknowledgement}

The author acknowledges the support of Dr. Karin Kaufman, the founder and President of Natural State Research, Inc (NSR). The author also acknowledges the valuable contributions NSR laboratory team members during the preparation of this manuscript.

\section{Reference}

[1] J. Aguado, ${ }^{*}$ D. P. Serrano, G. Vicente, and N. Sa'nchez, Enhanced Production of r-Olefins by Thermal Degradation of High-Density Polyethylene (HDPE) in Decalin Solvent: Effect of the Reaction Time and Temperature, Ind. Eng. Chem. Res. 2007, 46, 34973504

[2] Antonio Marcilla, * AÄ ngela N. Garcı́a, and Maria del Remedio Hernández, Thermal Degradation of LDPE-Vacuum Gas Oil Mixtures for Plastic Wastes Valorization, Energy \& Fuels 2007, 21, 870-880

[3] Achyut K. Panda a,b, ${ }^{*}$, R.K. Singh a,1, D.K. Mishra b,2, Thermolysis of waste plastics to liquid fuel A suitable method for plastic waste management and manufacture of value added products - A world prospective, Renewable and Sustainable Energy Reviews 14 (2010) 233-248

[4] N. Miskolczia, L. Barthaa, G. Dea'ka, B. Jo' verb , Thermal degradation of municipal plastic waste for production of fuel-like hydrocarbons, Polymer Degradation and Stability 86 (2004) 357-366

[5] Miguel Miranda a, ${ }^{*}$, Filomena Pinto a, I. Gulyurtlu a, I. Cabrita a, C.A. Nogueira a, Arlindo Matos b, Response surface methodology optimization applied to rubber tyre and plastic wastes thermal conversion, Fuel 89 (2010) 2217-2229

[6] M. Stelmachowski *, Thermal conversion of waste polyolefins to the mixture of hydrocarbons in the reactor with molten metal bed, Energy Conversion and Management 51 (2010) 2016-2024

[7] Karishma Gobin, George Manos*, Polymer degradation to fuels over microporous catalysts as a novel tertiary plastic recycling method, Polymer Degradation and Stability 83 (2004) 267-279

[8] Weibing Ding, Jing Liang, and Larry L. Anderson*, Hydrocracking and Hydroisomerization of High-Density Polyethylene and Waste Plastic over Zeolite and Silica-Alumina-Supported Ni and Ni-Mo Sulfides, Energy \& Fuels 1997, 11, 1219-1224

[9] Anthony Warren, Mahmoud El-Halwagi *, An economic study for the co-generation of liquid fuel and hydrogen from coal and municipal solid waste, Fuel Processing Technology 49 (1996) 157-166

[10] Wei-Chiang Huang a,b,c, Mao-Suan Huang c,d,1, Chiung-Fang Huang a,b,c, Chien-Chung Chen $\mathrm{c}, \mathrm{e}_{,}{ }^{*}$, Keng-Liang Ou c,e,f,**, Thermochemical conversion of polymer wastes into hydrocarbon fuels over various fluidizing cracking catalysts, Fuel 89 (2010) 2305-2316

[11] Valerio Cozzani, † Cristiano Nicolella, $\ddagger$ Mauro Rovatti, $\ddagger$ and Leonardo Tognotti*,, , Influence of Gas- Phase Reactions on the Product Yields Obtained in the Pyrolysis of Polyethylene, Ind. Eng. Chem. Res. 1997, 36, 342-348 
[12] A. Marcilla, ${ }^{*}$ M. I. Beltra'n, and R. Navarro, Evolution with the Temperature of the Compounds Obtained in the Catalytic Pyrolysis of Polyethylene over HUSY, Ind. Eng. Chem. Res. 2008, 47, 6896-6903

[13] Ma del Remedio Herna'ndez, AÄ ngela N. Garcı́a, Amparo Gómez, Javier Agullo', and Antonio Marcilla*, Effect of Residence Time on Volatile Products Obtained in the HDPE Pyrolysis in the Presence and Absence of HZSM-5, Ind. Eng. Chem. Res. 2006, 45, 8770-8778

[14] Levent Ballice,* Mithat $\mathrm{Yu}^{*}$ ksel, and Mehmet Sag`lam, Classification of Volatile Products from the Temperature-Programmed Pyrolysis of Low- and High-Density Polyethylene, Energy \& Fuels 1998, 12, 925-928

[15] Paula A. Costa,, , Filomena J. Pinto,† Ana. M. Ramos, $\ddagger$ Ibrahim K. Gulyurtlu, † Isabel A. Cabrita, $\dagger$ and Maria. S. Bernardoł, Kinetic Evaluation of the Pyrolysis of Polyethylene Waste, Energy \& Fuels 2007, 21, 2489-2498

[16] Biswanath Saha† and Aloke K. Ghoshal*, Hybrid Genetic Algorithm and Model-Free Coupled Direct Search Methods for Pyrolysis Kinetics of ZSM-5 Catalyzed Decomposition of Waste Low-Density Polyethylene, Ind. Eng. Chem. Res. 2007, 46, 5485-5492

[17] R. W. J. Westerhout, J. Waanders, J. A. M. Kuipers, * and W. P. M. van Swaaij, Recycling of Polyethene and Polypropene in a Novel Bench-Scale Rotating Cone Reactor by High-Temperature Pyrolysis, Ind. Eng. Chem. Res. 1998, 37, 2293-2300

[18] R. W. J. Westerhout, J. Waanders, J. A. M. Kuipers,* and W. P. M. van Swaaij, Development of a Continuous Rotating Cone Reactor Pilot Plant for the Pyrolysis of Polyethene and Polypropene, Ind. Eng. Chem. Res. 1998, 37, 2316-2322

[19] R. W. J. Westerhout, R. H. P. Balk, R. Meijer, J. A. M. Kuipers, ${ }^{*}$ and W. P. M. van Swaaij, Examination and Evaluation of the Use of Screen Heaters for the Measurement of the High Temperature Pyrolysis Kinetics of Polyethene and Polypropene, Ind. Eng. Chem. Res. 1997, 36, 3360-3368

[20] Lan Tang, * H. Huang, Zengli Zhao, C. Z. Wu, and Y. Chen, Pyrolysis of Polypropylene in a Nitrogen Plasma Reactor, Ind. Eng. Chem. Res. 2003, 42, 1145-1150

[21] George Manos, ${ }^{*}, \dagger$ Arthur Garforth, $\ddagger$ and John Dwyer§, Catalytic Degradation of HighDensity Polyethylene on an Ultrastable-Y Zeolite. Nature of Initial Polymer Reactions, Pattern of Formation of Gas and Liquid Products, and Temperature Effects, Ind. Eng. Chem. Res. 2000, 39, 1203-1208,

[22] George Manos, ${ }^{*}, \dagger$ Arthur Garforth, $\ddagger$ and John Dwyer\&, Catalytic Degradation of HighDensity Polyethylene over Different Zeolitic Structures, Ind. Eng. Chem. Res. 2000, 39, 1198-1202

[23] Yoshio Uemichi, * Junko Nakamura, Toshihiro Itoh, and Masatoshi Sugioka, Conversion of Polyethylene into Gasoline-Range Fuels by Two-Stage Catalytic Degradation Using Silica-Alumin and HZSM-5 Zeolite, Ind. Eng. Chem. Res. 1999, 38, 385-390

[24] George Manos, ${ }^{*}, \dagger$ Isman Y. Yusof, $\ddagger$ Nikos Papayannakos,§ and Nicolas H. Gangas§, Catalytic Cracking of Polyethylene over Clay Catalysts. Comparison with an Ultrastable Y Zeolite,Ind. Eng. Chem. Res. 2001, 40, 2220-2225

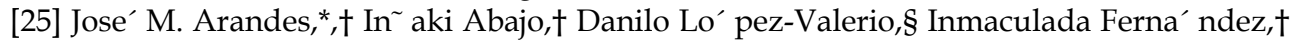
Miren J. Azkoiti, $\ddagger$ Martı́n Olazar, $†$ and Javier Bilbao†, Transformation of Several Plastic Wastes into Fuels by Catalytic Cracking, Ind. Eng. Chem. Res. 1997, 36, 45234529 
[26] Selhan Karago“"z,†,§ Jale Yanik, ${ }^{*}, \ddagger$ Suat Ucüar, $\dagger$ and Chunshan Song§, Catalytic Coprocessing of Low-Density Polyethylene with VGO Using Metal Supported on Activated Carbon, Energy \& Fuels 2002, 16, 1301-1308

[27] Toshiyuki Kanno, Masahiro Kimura, Na-oki Ikenaga, and Toshimitsu Suzuki*, Coliquefaction of Coal with Polyethylene Using Fe (CO) 5-S as Catalyst, Energy $\mathcal{E}$ Fuels 2000, 14, 612-617

[28] Mohammad Nahid Siddiqui a, ${ }^{*}$, Halim Hamid Redhwi b, Catalytic coprocessing of waste plastics and petroleum residue into liquid fuel oils, J. Anal. Appl. Pyrolysis 86 (2009) 141-147

[29] Ikusei Nakamura *, Kaoru Fujimoto, Development of new disposable catalyst for waste plastics treatment for high quality transportation fuel, Catalysis Today 27 (1996) 175-179

[30] A.G. Buekens *, H. Huang, Catalytic plastics cracking for recovery of gasoline-range hydrocarbons from municipal plastic wastes, Resources, Conservation and Recycling 23 (1998) 163-181 


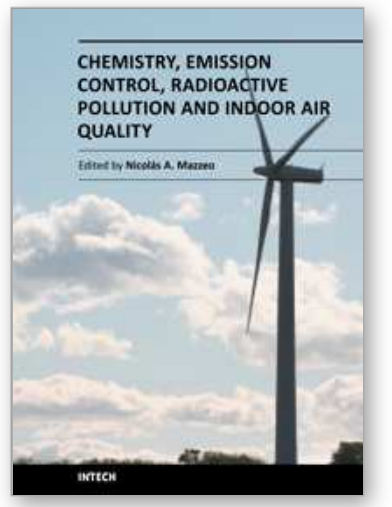

\author{
Chemistry, Emission Control, Radioactive Pollution and Indoor Air \\ Quality \\ Edited by Dr. Nicolas Mazzeo
}

ISBN 978-953-307-316-3

Hard cover, 680 pages

Publisher InTech

Published online 27, July, 2011

Published in print edition July, 2011

The atmosphere may be our most precious resource. Accordingly, the balance between its use and protection is a high priority for our civilization. While many of us would consider air pollution to be an issue that the modern world has resolved to a greater extent, it still appears to have considerable influence on the global environment. In many countries with ambitious economic growth targets the acceptable levels of air pollution have been transgressed. Serious respiratory disease related problems have been identified with both indoor and outdoor pollution throughout the world. The 25 chapters of this book deal with several air pollution issues grouped into the following sections: a) air pollution chemistry; b) air pollutant emission control; c) radioactive pollution and d) indoor air quality.

\title{
How to reference
}

In order to correctly reference this scholarly work, feel free to copy and paste the following:

Moinuddin Sarker (2011). Municipal Waste Plastic conversion into Different Category Liquid Hydrocarbon Fuel, Chemistry, Emission Control, Radioactive Pollution and Indoor Air Quality, Dr. Nicolas Mazzeo (Ed.), ISBN: 978-953-307-316-3, InTech, Available from: http://www.intechopen.com/books/chemistry-emission-controlradioactive-pollution-and-indoor-air-quality/municipal-waste-plastic-conversion-into-different-category-liquidhydrocarbon-fuel

\section{INTECH}

open science | open minds

\author{
InTech Europe \\ University Campus STeP Ri \\ Slavka Krautzeka 83/A \\ 51000 Rijeka, Croatia \\ Phone: +385 (51) 770447 \\ Fax: +385 (51) 686166 \\ www.intechopen.com
}

\author{
InTech China \\ Unit 405, Office Block, Hotel Equatorial Shanghai \\ No.65, Yan An Road (West), Shanghai, 200040, China \\ 中国上海市延安西路65号上海国际贵都大饭店办公楼405单元 \\ Phone: +86-21-62489820 \\ Fax: +86-21-62489821
}


(C) 2011 The Author(s). Licensee IntechOpen. This chapter is distributed under the terms of the Creative Commons Attribution-NonCommercialShareAlike-3.0 License, which permits use, distribution and reproduction for non-commercial purposes, provided the original is properly cited and derivative works building on this content are distributed under the same license. 